\title{
The hepatic WASH complex is required for efficient plasma LDL and HDL cholesterol clearance
}

Melinde Wijers, ${ }^{1}$ Paolo Zanoni, ${ }^{2}$ Nalan Liv, ${ }^{3}$ Dyonne Y. Vos, ${ }^{1}$ Michelle Y. Jäckstein, ${ }^{1}$ Marieke Smit, ${ }^{1}$ Sanne Wilbrink, ${ }^{1}$ Justina C. Wolters, ${ }^{1}$ Ydwine T. van der Veen, ${ }^{1}$ Nicolette Huijkman, ${ }^{1}$ Daphne Dekker, ${ }^{1}$ Niels Kloosterhuis, ${ }^{1}$ Theo H. van Dijk ${ }^{4}$ Daniel D. Billadeau, ${ }^{5}$ Folkert Kuipers ${ }^{1,4}$ Judith Klumperman, ${ }^{3}$ Arnold von Eckardstein, ${ }^{2}$ Jan Albert Kuivenhoven, ${ }^{1}$ and Bart van de Sluis ${ }^{1}$

'Department of Pediatrics, University of Groningen, University Medical Center Groningen, Groningen, Netherlands. ${ }^{2}$ Institute for Clinical Chemistry, University Hospital Zurich, Zurich, Switzerland; Centre for Integrative Human Physiology, University of Zurich, Zurich, Switzerland. ${ }^{3}$ Section Cell Biology, Center for Molecular Medicine, University Medical Center Utrecht, Utrecht University, Utrecht, Netherlands. ${ }^{4}$ Laboratory Medicine, University of Groningen, University Medical Center Groningen, Groningen, Netherlands. ${ }^{5}$ Department of Immunology and Biochemistry, Division of Oncology Research, Mayo Clinic, Rochester, New York, USA.

The evolutionary conserved Wiskott-Aldrich syndrome protein and SCAR homolog (WASH) complex is one of the crucial multiprotein complexes that facilitates endosomal recycling of transmembrane proteins. Defects in WASH components have been associated with inherited developmental and neurological disorders in humans. Here, we show that hepatic ablation of the WASH component Washc1 in chow-fed mice increases plasma concentrations of cholesterol in both LDLs and HDLs, without affecting hepatic cholesterol content, hepatic cholesterol synthesis, biliary cholesterol excretion, or hepatic bile acid metabolism. Elevated plasma LDL cholesterol was related to reduced hepatocytic surface levels of the LDL receptor (LDLR) and the LDLR-related protein LRP1. Hepatic WASH ablation also reduced the surface levels of scavenger receptor class B type I and, concomitantly, selective uptake of HDL cholesterol into the liver. Furthermore, we found that WASHC1 deficiency increases LDLR proteolysis by the inducible degrader of LDLR, but does not affect proprotein convertase subtilisin/kexin type 9-mediated LDLR degradation. Remarkably, however, loss of hepatic WASHC1 may sensitize LRP1 for proprotein convertase subtilisin/kexin type 9-induced degradation. Altogether, these findings identify the WASH complex as a regulator of LDL as well as HDL metabolism and provide in vivo evidence for endosomal trafficking of scavenger receptor class B type I in hepatocytes.

Authorship note: PZ, NL, DYV, and MYJ contributed equally to this work.

Conflict of interest: The authors have declared that no conflict of interest exists.

Copyright: (c) 2019 American Society for Clinical Investigation

Submitted: December 5, 2018 Accepted: April 17, 2019 Published: June 6, 2019.

Reference information: /CI Insight. 2019;4(11):e126462. https://doi. org/10.1172/jii.insight.126462

\section{Introduction}

The endosomal network plays a crucial role in the delivery of integral membrane proteins (also referred to as cargo proteins) either to lysosomes for proteolysis, to the trans-Golgi network, or to the plasma membrane. In recent years, the Wiskott-Aldrich syndrome protein and SCAR homolog (WASH) complex has emerged as one of the key multiprotein complexes facilitating the endosomal sorting of cargos (1-3), such as the EGFR (4), copper-transporting P-type ATPase ATP7A (5), and the $\beta 2$-adrenoreceptor $(6,7)$. The WASH complex consists of 5 subunits, WASHC1 (WASH1), WASHC2 (FAM21), WASHC3 (CCDC53), WASHC4 (SWIP), and WASHC5 (Strumpellin or KIAA0196) and governs the destination of cargos by activating the Arp $2 / 3$ complex $(8,9)$. Arp $2 / 3$ forms actin-enriched subdomains on the endosomal membrane from where cargos are sorted and transported $(10,11)$.

Mutations in several components of the WASH complex have been identified in developmental and neurological disorders: mutations in WASHC4 have been found in patients with nonsyndromic autosomal recessive intellectual disability (12), while defects in WASHC5 have been reported in patients with autosomal dominant hereditary spastic paraplegia $(13,14)$ or Ritscher-Schinzel/3C syndrome (RSS) (15). Surprisingly, our recent in vitro work also implicated a role for the WASH complex in the metabolism of LDL cholesterol (LDL-C). We found that WASHC1 deficiency impairs endosomal trafficking of the LDL receptor 
(LDLR), leading to LDLR accumulation in endosomes, reduced LDLR surface levels and impaired LDL uptake in mouse embryonic fibroblasts (MEFs) (16). Furthermore, we showed that hepatic destabilization of the WASH-associated CCC (COMMD-CCDC22-CCDC93) complex increases plasma LDL-C levels in mice, dogs, and humans and accelerates atherosclerosis in mice $(16,17)$. These data strongly indicate the involvement of the WASH complex in the regulation of cholesterol homoeostasis. Accordingly, we reported a patient with familial RSS presenting with hypercholesterolemia (16), beyond developmental malformation and intellectual disability. However, due to the clinical complexity of RSS and because we assessed the plasma cholesterol levels from only 1 patient with RSS, the true contribution of the WASH complex to cholesterol homeostasis at an organismal level has remained unclear. Furthermore, it has been shown that the endolysosomal system is essential for the proprotein convertase subtilisin-kexin type 9 (PCSK9) and inducible degrader of LDLR (IDOL)-mediated LDLR degradation pathways (18-22), but the role of the WASH-CCC axis in these 2 pathways is still unknown.

We, therefore generated liver-specific WASH-deficient mice to establish the role of the WASH complex in the regulation of whole-body cholesterol metabolism. We found that ablation of the WASH complex leads to elevated plasma levels of cholesterol in both LDL and HDL fractions. In addition, we observed that WASH is not only required for the cell surface expression of the LDLR family members (i.e., LDLR and LDLR-related protein 1 [LRP1]), but also of the scavenger receptor class B type I (SR-BI), the main receptor that mediates selective uptake of cholesteryl esters (CEs) from HDL into hepatocytes. Kinetic studies showed that selective HDL-CE uptake was impaired in hepatic WASH-deficient mice. Finally, we found that the WASH complex delays IDOL-mediated LDLR degradation but does not influence PCSK9-induced LDLR proteolysis. Intriguingly, hepatic Washc1 ablation sensitized LRP1 degradation by PCSK9 in vivo. Altogether, our results imply that, in addition to its role in developmental and neurological processes, the WASH complex is involved in hepatic uptake of plasma LDL-C, HDL cholesterol (HDL-C), and IDOL-mediated LDLR degradation.

\section{Results}

Depletion of Washc1 destabilizes the WASH complex and adversely affects the lysosomal architecture in hepatocytes. To decipher the role of the hepatic WASH complex in cholesterol homeostasis, we generated a liver-specific Washc1-knockout model (Washc1 ${ }^{\Delta \mathrm{Hep}}$ ) by cross-breeding mice carrying floxed Washc1 alleles (Washc1 $1^{\text {flff }}$ ) (4) with transgenic mice expressing Cre recombinase in hepatocytes under the control of the Albumin (Alb) gene promoter. Successful ablation of hepatic WASHC1 was confirmed by immunoblotting (Figure 1, A and B). In line with previous findings in MEFs (4), hepatic WASHC1 deficiency strongly reduced the abundance of all WASH components (Figure 1, A and B) without affecting the mRNA levels of these subunits (Supplemental Figure 1A; supplemental material available online with this article; https://doi.org/10.1172/jci. insight.126462DS1), illustrating the importance of WASHC1 in the formation of a stable WASH complex in mouse liver. Washc1 $1^{\triangle \mathrm{Hep}}$ mice were born at the expected Mendelian ratio (Supplemental Figure 1B) and showed no overt changes in body weight, liver weight, or general liver histology compared with littermate controls (from now on referred to as WT) (Supplemental Figure 1, C and D). WASH binds to many different multiprotein complexes, such as the CCC complex, retriever, and retromer, to coordinate the endosomal trafficking of cargos $(5,9,16,23-26)$. Ablation of the WASH complex did not have a large effect on the abundance of any subunit of these complexes (Supplemental Figure 1E).

In vitro studies have shown that WASH deficiency alters the morphology of the endosomes and lysosomal structure $(4,8-10)$. We therefore examined the lysosomal structure by electron microscopy (EM) in liver sections of WT and Washc $1^{\Delta \mathrm{Hep}}$ mice. Hepatic ablation of the WASH complex decreased the average size of the lysosomes (Figure 1, C and D). In addition, we found that WASH deficiency induced lysosomal tubulation (Figure 1E). In WT hepatocytes, 4.4\% (11 of 250 lysosomes) of the lysosomes were tubulated, whereas in Washc1 ablated hepatocytes, the percentage of tubulated lysosomes was increased to $14.8 \%$ (37 of 250 lysosomes). However, the loss of hepatic WASH did not noticeably alter the morphology of other organelles such as Golgi, endoplasmic reticulum, or mitochondria. These results demonstrate that we successfully deleted Washcl in mouse livers, which results in destabilization of the WASH complex and changes in lysosomal architecture in mouse hepatocytes in vivo.

Loss of hepatic WASH results in elevated plasma LDL and HDL-C levels. To determine the effect of hepatic WASH deficiency on circulating lipids, we measured total plasma cholesterol (TC) and triglyceride (TG) levels in chow-fed WT and Washc1 ${ }^{\Delta \mathrm{Hep}}$ mice. Hepatic inactivation of the WASH complex increased TC without affecting TG levels (Figure 2A). Using targeted proteomics (17), we observed increased plasma 
levels of apolipoproteins $\mathrm{ApoE}$ and $\mathrm{ApoB}$ in Washc $1^{\mathrm{\Delta Hep}}$ mice compared with WT mice (Figure 2B). In line with the increased ApoB levels, the cholesterol content of the LDL fraction separated by fast protein liquid chromatography (FPLC) was increased (Figure 2, C and D). We also found the HDL fraction to be enriched with cholesterol and increased in size (Figure 2C). Although total plasma ApoA-I levels were not affected (Figure 2B) in Washc $1^{\mathrm{HHep}}$ mice, we observed a shift of ApoA-I toward earlier eluting FPLC fractions (Figure 2E). These fractions also contain ApoE (Figure 2F). Altogether, the shift of ApoA-I, ApoE, and HDL-C into earlier eluted FPLC fractions indicates that HDL particles in Wash $^{4{ }^{\mathrm{Hep}}}$ mice are larger than in WT mice (27-29). An increase in TC, LDL-C, and larger HDL particles was also seen in female Washc1 $1^{\text {Hep }}$ mice (Supplemental Figure 2, A-C).

In contrast to humans, mice do not express cholesteryl ester transfer protein (CETP), which exchanges HDL-C esters for TG in very low-density lipoprotein (VLDL) and LDL (30). As a consequence, mice carry plasma cholesterol mainly in HDL rather than in LDL (31). This modest plasma LDL-C increase in Washc1 ${ }^{\Delta \mathrm{Hep}}$ mice is very similar as seen in mice heterozygous for an Ldlr-knockout allele (32). To further assess the effect of hepatic Washc1 deletion on plasma LDL and HDL-C levels in mice with a more human-like lipoprotein profile, we injected both $W a s h c 1^{\Delta \mathrm{Hep}}$ and WT mice with an adenovirus-expressing human CETP (Ad-CETP). A similar hepatic level of human CETP expression was observed in Washcl $^{\Delta \mathrm{Hep}}$ and WT mice (Figure 3A). In agreement with previous studies $(33,34)$, ectopic expression of CETP decreased TC levels in mice (Figure 3B) compared with mice not expressing CETP (Figure 2A), but the TC levels were significantly higher in CETP-expressing Washc1 ${ }^{\Delta \mathrm{Hep}}$ mice compared with WT mice (Figure 3B). Hepatic expression of human CETP resulted in the expected shift of cholesterol from HDL toward LDL in both $\mathrm{Wash}_{\mathrm{c}} 1^{\mathrm{\Delta Hep}}$ and WT mice (Figure 3C). However, in Washc $1^{\Delta \text { Hep }}$, the LDL-C, HDL-C, as well as the apolipoproteins ApoB, Apo-AI, and ApoE levels were increased compared with WT mice (Figure 3, C and D). CETP expression normalized the increased HDL particles in the Washc1 $1^{\Delta \mathrm{Hep}}$ mice (Figure 2C) compared with Washc1 ${ }^{\Delta \mathrm{Hep}}$ mice without CETP expression (Figure 3C), a phenomenon also seen in SR-B1-deficient mice upon CETP expression (34).

We found that hepatic Washc1 ablation did not affect hepatic cholesterol and TG contents, VLDL production, and whole-body cholesterol synthesis rates (Supplemental Figure 3, A-D). Several liver X receptor (LXR) targets, including genes involved in biliary cholesterol secretion and bile acid synthesis ( $A b c g 5$, $A b c g 8$, and Cyp7a1) were decreased upon hepatic Washc1 depletion compared to WT livers (Supplemental Table 1), suggesting that LXR activation was decreased in Washc $1^{\Delta \mathrm{Hep}}$ mice. However, these changes did not translate into aberrant biliary cholesterol and bile acid excretion nor in altered fecal neutral sterol and bile acid loss (Supplemental Figure 3, E-H). No significant differences in the mRNA levels of the SREBP-2 target genes, such as Ldlr and Hmgcr, were found (Supplemental Table 1).

These data indicate that the WASH complex in the liver plays an important role in the regulation of plasma LDL-C and HDL-C levels, but does not have a prominent role in the control of whole-body cholesterol turnover.

The WASH complex is required for normal expression of LDLR and LRP1 at the cell surface. We have recently shown that WASH mediates endosomal recycling of LDLR back to the cell surface in MEFs (16). Therefore, we hypothesized that the increased LDL-C levels in Washc1 $1^{\Delta \mathrm{Hep}}$ are due to reduced surface levels of LDLR caused by impaired LDLR recycling. To test this theory, cell surface levels of LDLR were determined in primary hepatocytes using a biotinylation assay. We found that the levels of LDLR were reduced in the plasma membranes of WASH-deficient primary hepatocytes compared with WT cells (Figure 4, A and B). Previously, we showed that the WASH-associated CCC complex also facilities the trafficking of LRP1 to the plasma membrane of primary hepatocytes (17). Consistent with this finding, we learned that WASH is also required for normal levels of LRP1 at the cell surface (Figure 4, A and B). In addition to the surface levels, the total protein levels of LDLR and LRP1 were also reduced in WASH-deficient primary hepatocytes (Figure 4, A and B). This is likely due to the impaired retrieval of these receptors from the lysosomal proteolytic fate into the recycling pathway $(4,16,23)$.

In mice, the role of hepatic LRP1 in the clearance of plasma cholesterol is redundant, as LDLR can compensate for LRP1 loss (35). To address the role of WASH in LRP1-mediated plasma cholesterol uptake, we expressed a human gain-of-function variant of PCSK9 (D377Y) in mouse livers using an adeno-associated virus (AAV) expression system to ablate hepatic LDLR levels $(17,36)$. In both Washc $^{4 \text { Hep }}$ and WT mice, the expression of PCSK9-D377Y blunted hepatic LDLR levels (Figure 4C). Remarkably, although PCSK9 does not have an effect on hepatic LRP1 degradation in WT mice $(17,37,38)$, we found that in the absence 
A

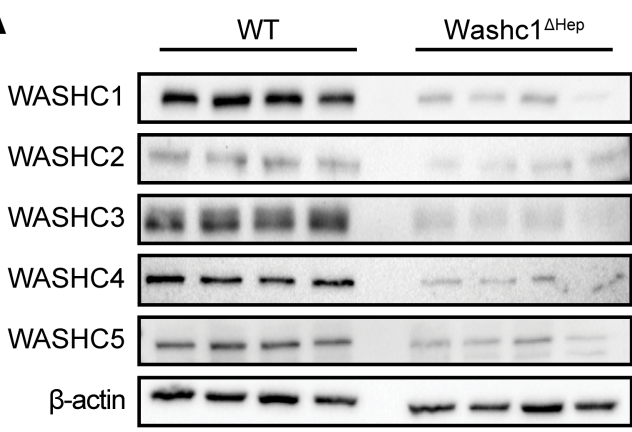

C

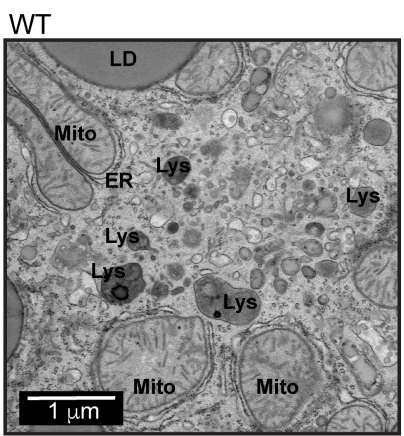

Washc $1^{\Delta \mathrm{Hep}}$

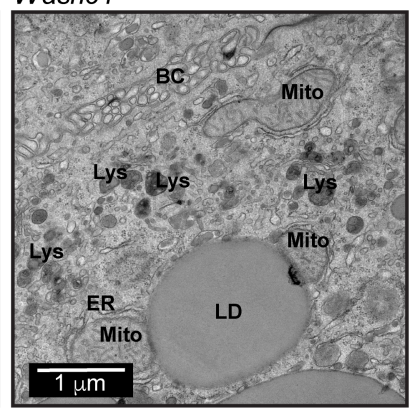

$\mathbf{E}$
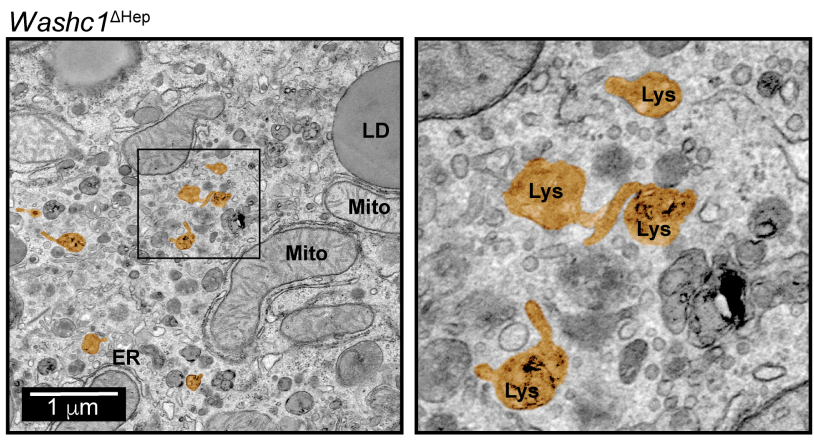

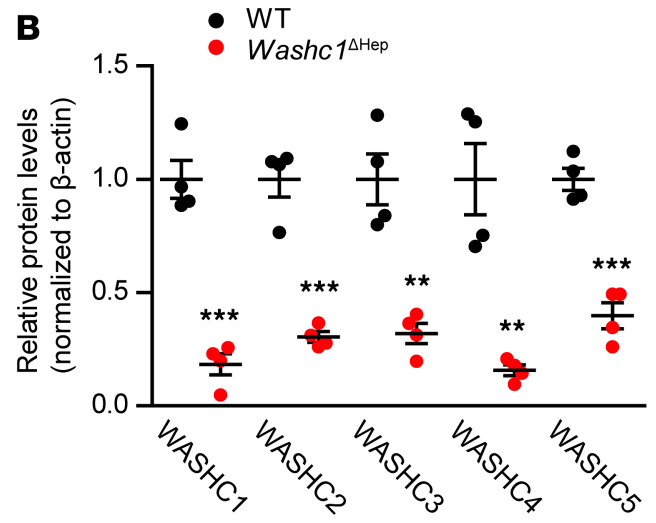

D

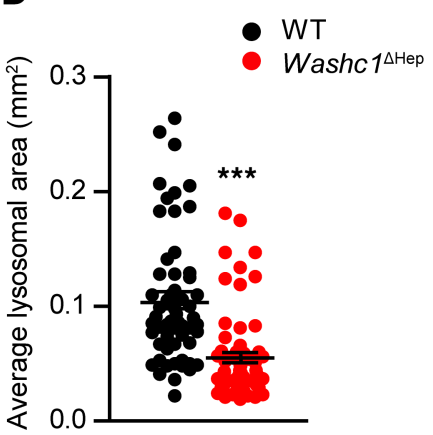

Figure 1. WASHC1 deficiency destabilizes the WASH complex and perturbs lysosomal structures in primary hepatocytes. (A) Protein expression of the WASH complex components in liver homogenates of WT and Washc ${ }^{\text {Hep }}$ mice as determined by immunoblotting. (B) Quantification of immunoblot results depicted in A. (C) EM images of WT and Washc1 1Нер livers. BC, bile canaliculus, ER, endoplasmic reticulum; LD, lipid droplet; Lys, lysosome; Mito, mitochondrion. (D) Quantification of WT $(n=57)$ and Washc ${ }^{\text {HHep }}(n=63)$ lysosomal area in liver sections. (E) EM image depicting aberrant lysosomal structure in Washc $7^{1 \mathrm{Hep}}$ hepatocyte. Inset shows magnified area with tubulated lysosomes. For visualization of lysosomal tubulation, lysosomal perimeters were selected using the Lasso tool in Adobe Photoshop and color was added to the selected organelles. Data are presented as the mean $\pm \mathrm{SEM},{ }^{*} P<0.05$, ${ }^{* *} P<0.01,{ }^{* *} P<0.001$ as determined by Student's $t$ test.

of the WASH complex, LRP1 levels were significantly decreased upon PCSK9 overexpression in mouse livers (Figure 4, C and D, and Supplemental Figure 4). As shown in Figure 4E, this modest decrease in LRP1 levels was not caused by a decrease in Lrp1 mRNA levels.

We found that hepatic Washc1 depletion exacerbated hypercholesterolemia in PCSK9-induced hepatic LDLR deficiency (Figure 4F). Consistent with the increased plasma chylomicron remnant/VLDL and LDL lipoprotein fractions (Figure 4G), plasma ApoB48 and ApoB100 levels were elevated in mice deficient for hepatic WASH and LDLR compared with mice lacking only hepatic LDLR (Figure 4, H and I). Hepatic expression of PCSK9 also increased ApoA-I levels in this model, while HDL-C levels were unchanged (Figure 4H). Hepatic WASH/LDLR deficiency mimics the plasma lipid phenotype in hepatic LRP1-deficient mice in an Ldlr-knockout background (35), suggesting that the WASH complex is also required for hepatic LRP1-mediated cholesterol uptake in vivo. These data demonstrate that hepatic 
A

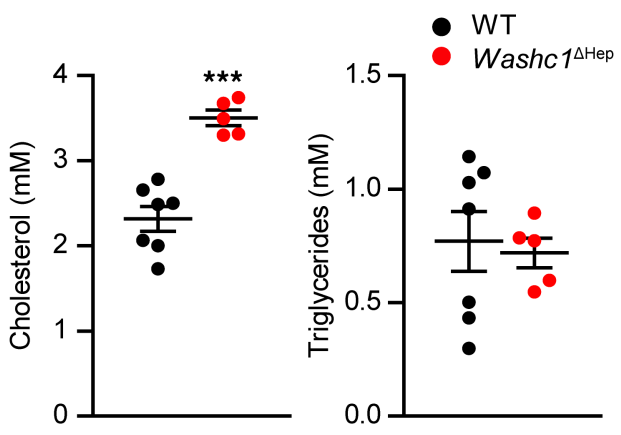

C

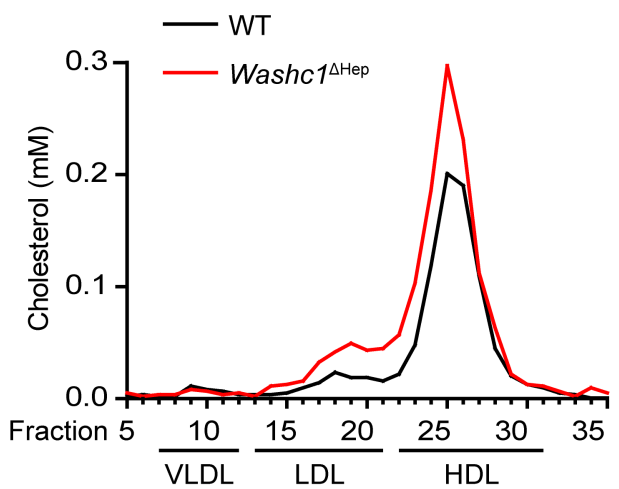

$\mathbf{E}$

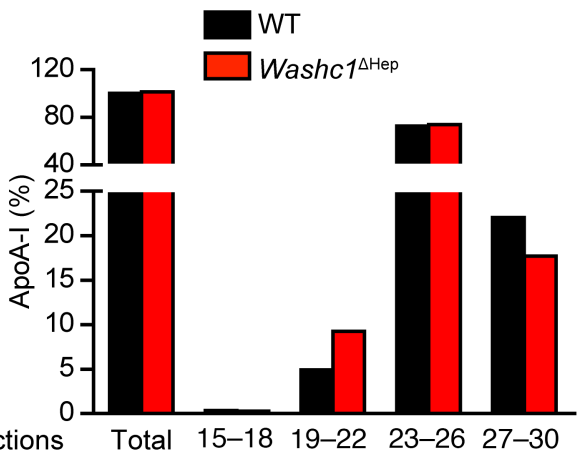

B

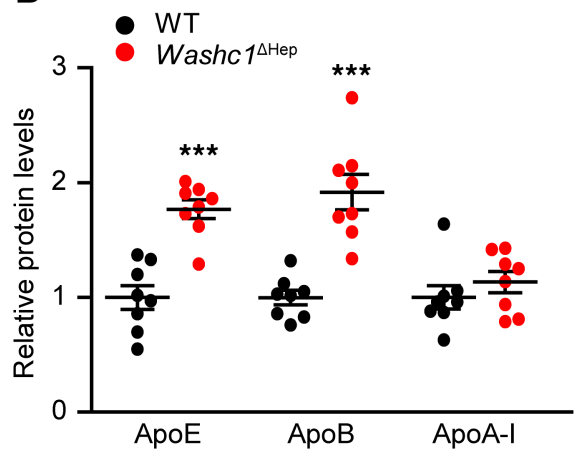

D

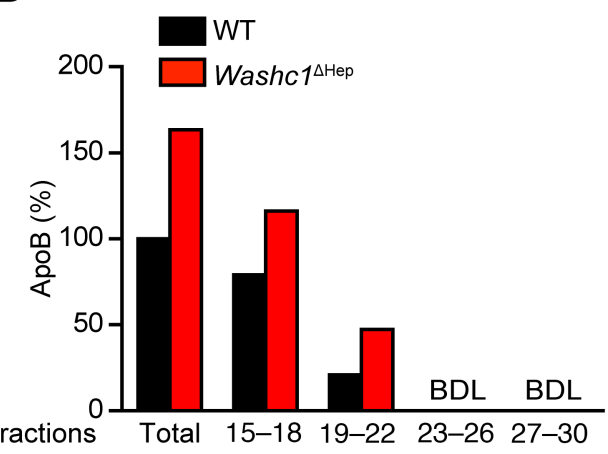

$\mathbf{F}$

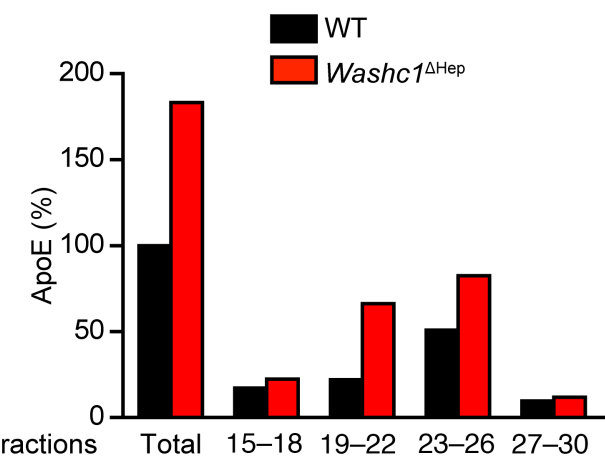

Figure 2. Loss of hepatic WASHC1 results in increased plasma LDL-C and HDL-C. (A) Total plasma cholesterol and TC levels in WT and Washc1 ${ }^{\text {Hep }}$ male mice $(n=5-7)$. (B) Plasma apolipoprotein levels in WT and Washc1 ${ }^{1 \text { Hep }}$ mice measured by targeted proteomics, indicated by fold change vs WT controls $(n=8)$. (C) Cholesterol levels in FPLC fractionated plasma pools of the experimental groups of mice. (D-F) Apolipoproteins in pooled FPLC fractionated plasma of WT and Washc1 ${ }^{\text {Hep }}$ mice depicted in $\mathbf{C}$ determined by targeted proteomics, indicated as percentage relative to the total apolipoprotein levels in these fractions $(n=5-7)$. BDL, below detection limit. Data are presented as the mean \pm SEM, ${ }^{*} P<0.05,{ }^{* *} P<0.01,{ }^{* *} P<0.001$ as determined by Student's $t$ test.

depletion of WASH impairs the functioning of both LDLR and LRP1 in the uptake of plasma cholesterol leading to hypercholesterolemia and, likely, sensitizes LRP1 for PCSK9-induced degradation.

HDL-C uptake is impaired in hepatic WASH-deficient mice due to reduced surface SR-BI levels. In addition to accumulation of cholesterol in LDL, we also found plasma levels of HDL-C to be increased in Washc $1^{\text {AHep }}$ mice (Figure 2C). In mice, HDL-C is mainly cleared by SR-BI, a membrane receptor that selectively takes up CE from HDL rather than internalizing the holoparticle (39). To assess whether the accumulation of plasma HDL-C in Washc $1^{\text {Hep }}$ mice is due to impaired endosomal trafficking of SR-BI to the plasma membrane, we applied a biotinylation assay to examine the cell surface levels of SR-BI in primary hepatocytes. We found the cell surface abundance of SR-BI to be markedly decreased in WASH-deficient hepatocytes compared with WT cells (Figure 5A). It has been shown that the enzyme hepatic lipase (HL) increases 
A

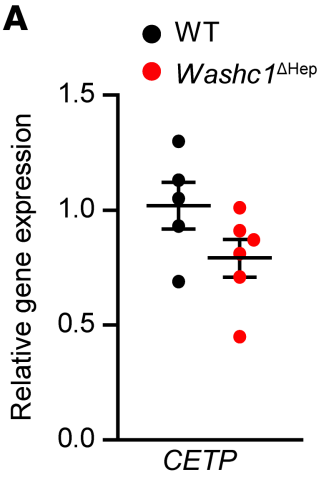

B

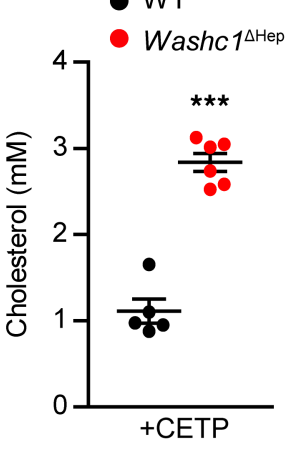

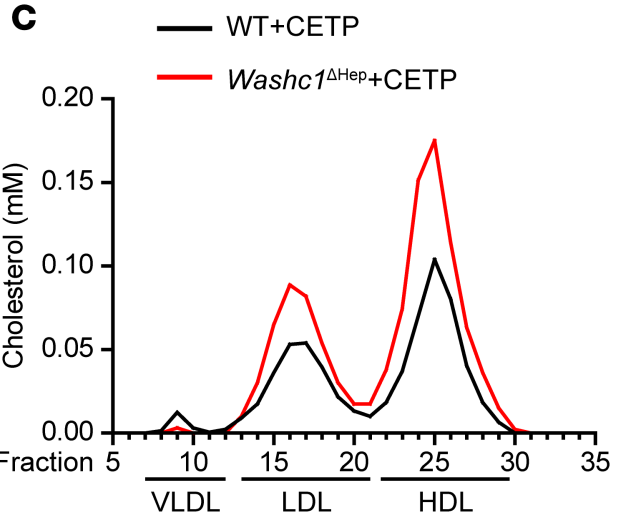

D

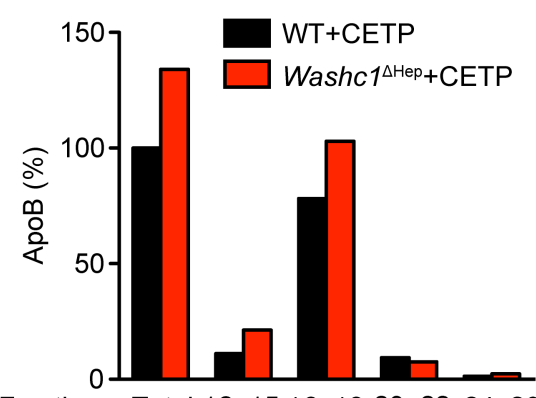

Fractions Total 12-15 16-19 20-23 24-30

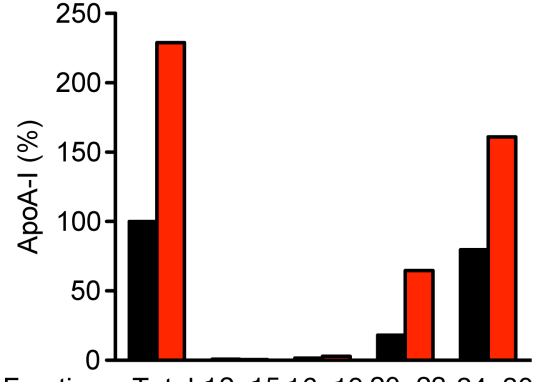

Fractions Total 12-15 16-19 20-23 24-30

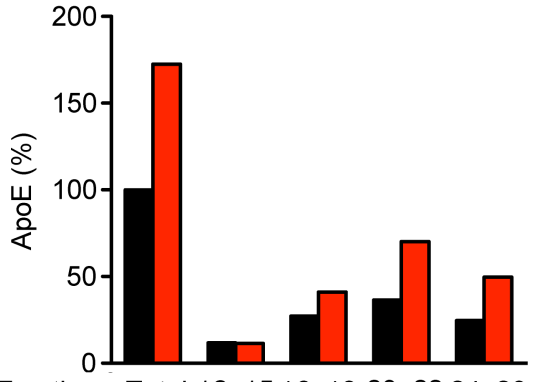

Fractions Total 12-15 16-19 20-23 24-30

Figure 3. Hepatic WASHC1 deficiency results in increased plasma LDL-C and HDL-C in mice expressing CETP. (A) Hepatic CETP mRNA expression after Ad-CETP administration. (B) Total plasma cholesterol levels 3 days after Ad-CETP administration in male WT and Washc1 ${ }^{\text {HHep }}$ mice. (C) Cholesterol levels of FPLC fractionated pooled plasma of the experimental groups of mice after Ad-CETP administration $(n=5-6)$. (D) Apolipoproteins in pooled FPLC fractionated plasma of WT + CETP and Washc1 1Hep + CETP mice depicted in C determined by targeted proteomics, indicated as percentage relative to the total apolipoprotein levels in these fractions. Data are presented as the mean \pm SEM, ${ }^{* * *} P<0.001$ as determined by Student's $t$ test.

hepatic HDL-CE uptake (40-43). However, we did not find any difference in plasma HL levels as measured by targeted proteomics (Figure $5 \mathrm{~B}$ ), which makes it unlikely that HL contributes to the increased plasma HDL-C levels in hepatic WASH-deficient mice.

To establish the consequences of the reduced hepatocyte SR-BI surface levels in hepatic WASH-deficient mice in more detail, we compared the uptake of HDL-associated CE and HDL holoparticles between WT and Washc $1^{\Delta \mathrm{Hep}}$ mice upon injecting these mice with HDL particles containing ${ }^{3} \mathrm{H}$-labeled CE as well as ${ }^{125} \mathrm{I}$-labeled proteins. Selective HDL-C uptake was calculated from the difference in the uptake of both tracers. Plasma decay of radioiodine reflecting holoparticle HDL was not different between WT and Washc ${ }^{1 \mathrm{Hep}}$ mice (Figure 5C). However, ${ }^{3} \mathrm{H}$-HDL-CE clearance was delayed upon hepatic WASH inactivation (Figure 5C). From the decay curves, we calculated plasma fractional catabolic rates and observed that HDL-CE clearance in hepatic WASH-deficient mice was significantly reduced compared with WT mice, whereas holoparticle uptake was not changed (Figure 5D). As a consequence, the difference reflecting selective HDL-CE uptake by Washc1 ${ }^{1 \mathrm{Hep}}$ livers was significantly decreased compared with WT livers (Figure 5E). The kidney is the main organ for the catabolism of small, poorly lipidated ApoA-I molecules and does not express SR-BI $(39,44)$. In line with previous studies (45), the renal uptake rate of HDL-CE was much lower than with hepatic HDL-CE uptake, and no differences were observed between WT and Washc $1^{\text {HHep }}$ mice (data not shown). Overall, these results indicate that the hepatic uptake of HDL-CE is impaired in Washc1 ${ }^{1 \mathrm{Hep}}$ mice likely due to reduced expression of SR-BI at the plasma membrane of hepatocytes.

Results from previous and current studies imply that the WASH and CCC complex act in concert to regulate plasma lipid levels. To validate this at an organismal level, we inactivated both protein complexes in mouse livers by cross-breeding Washc $1^{\Delta \mathrm{Hep}}$ mice with mice carrying Commd1 floxed alleles (Washc1/Commd1 $\left.{ }^{\Delta \mathrm{Hep}}\right)(16$, 46) (Figure 6A). Hepatic ablation of both complexes did not further increase total plasma cholesterol levels when compared with hepatic Washc1 and Commd1 single-knockout mice (Figure 6B). In addition, hepatic ablation of both complexes also resulted in increased plasma LDL-C (Figure 6, C and D) and larger HDL-C particles (Figure 6, C and D), similar to that was seen in single-knockout models (Figure 2 and refs. 16, 17). 
A

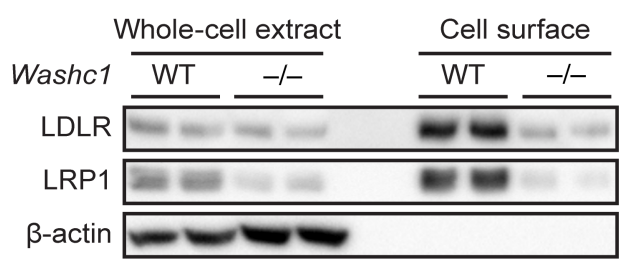

\section{B}
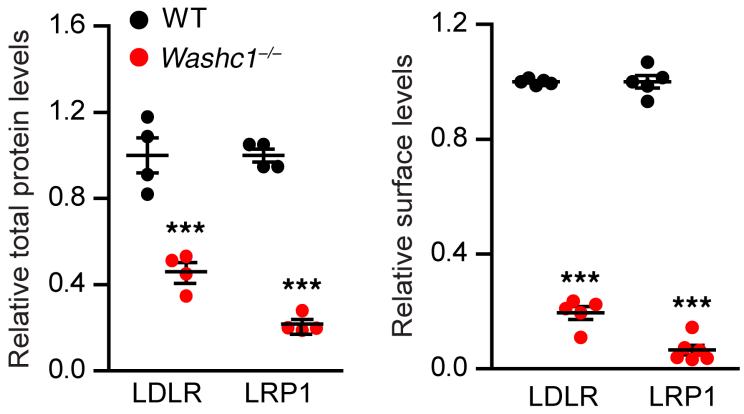

C

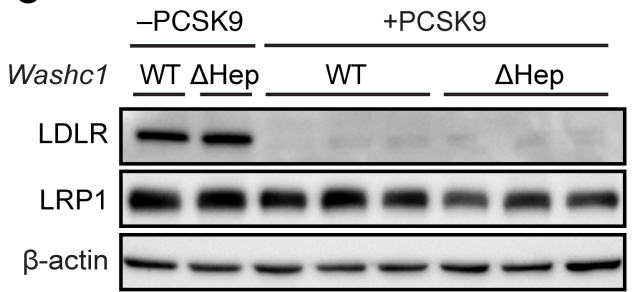

D

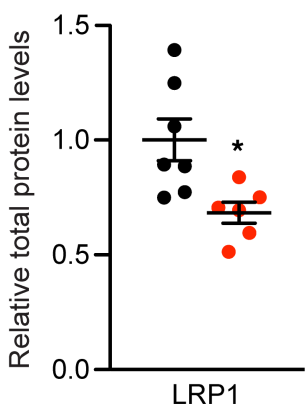

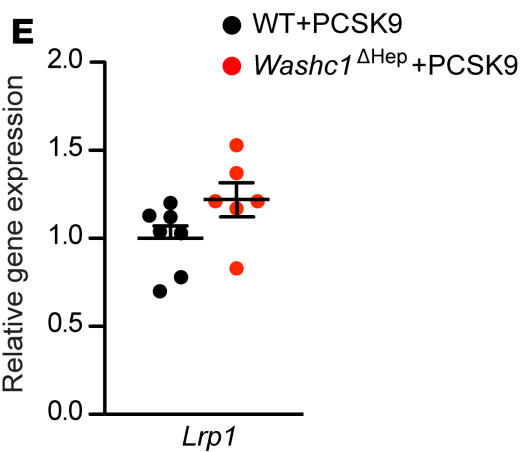

F

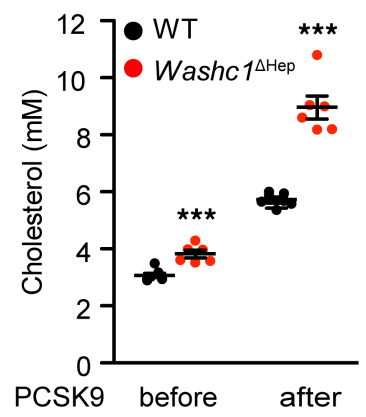

G

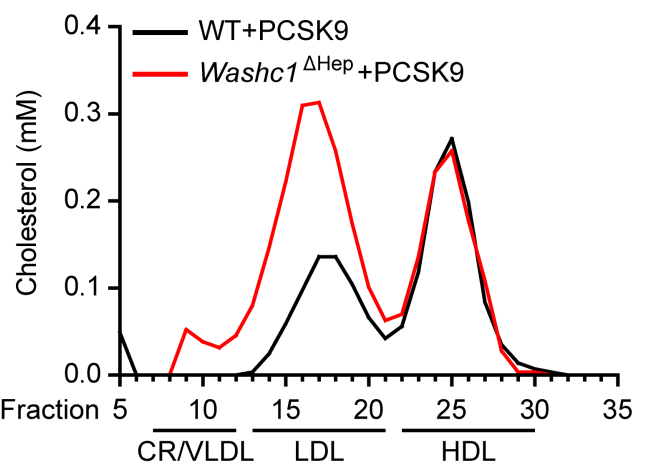

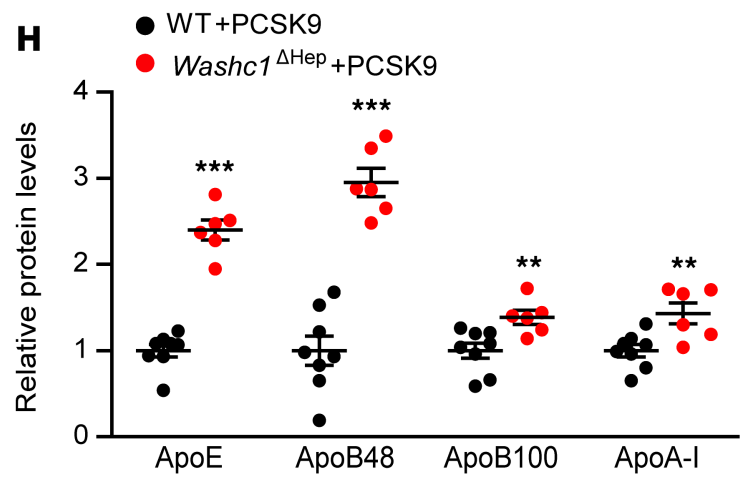

I

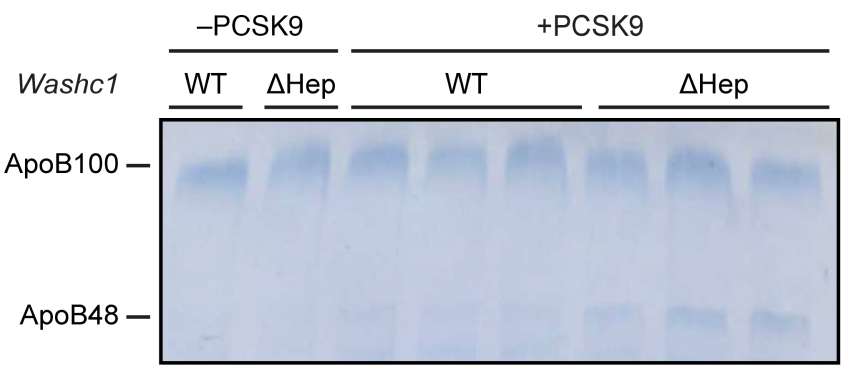

Figure 4. The WASH complex is required for normal cell surface expression of LDLR and LRP1. (A) Representative immunoblot of whole-cell and cell-surface protein levels of LDLR and LRP1 in WT and Washc1 ${ }^{-1-}$ primary hepatocytes. (B) Quantification of total and cell surface levels of LDLR and LRP1 ( $\left.n=4\right)$. (C) Representative immunoblot of hepatic LDLR and LRP1 3 weeks after AAV-PCSK9-D377Y administration. (D) Quantification of hepatic LRP1 protein levels in male WT and Washc1 1Hep mice 3 weeks after AAV-PCSK9-D377Y administration as determined by immunoblotting $(n=6-7)$. (E) Hepatic Lrp1 mRNA expression in the experimental groups of mice depicted in D. (F) Total plasma cholesterol levels in male WT and Washc1 ${ }^{\text {HHep }}$ mice before and 3 weeks after AAV-PCSK9D377Y administration ( $n=6-7)$. (C) TC levels of FPLC fractionated pooled plasma of WT and Washc1 ${ }^{\text {Hep }} 3$ weeks after AAV-PCSK9-D377Y administration. CR, chylomicron remnants $(n=6-7)$. (H) Relative plasma apolipoprotein levels in WT and Washc $7^{\text {1Hep }}$ mice after AAV-PCSK9-D377Y administration as measured by targeted proteomics. (I) Coomassie blue staining of ApoB100 and ApoB48 in WT and Washc1 1Hep plasma before and after AAV-PCSK9-D377Y administration. Data are presented as the mean $\pm \mathrm{SEM},{ }^{* *} P<0.01,{ }^{* * *} P<0.001$ as determined by Student's $t$ test. 
A

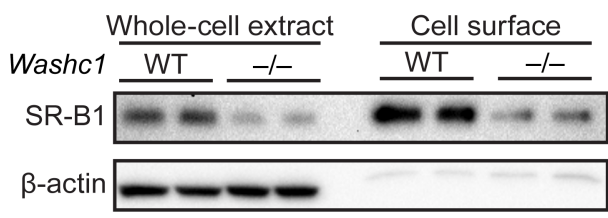

C

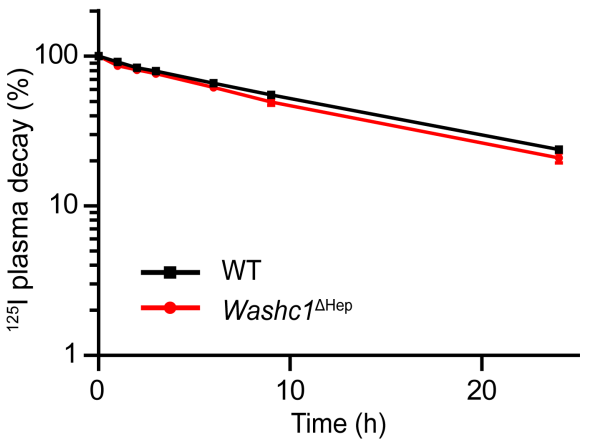

D

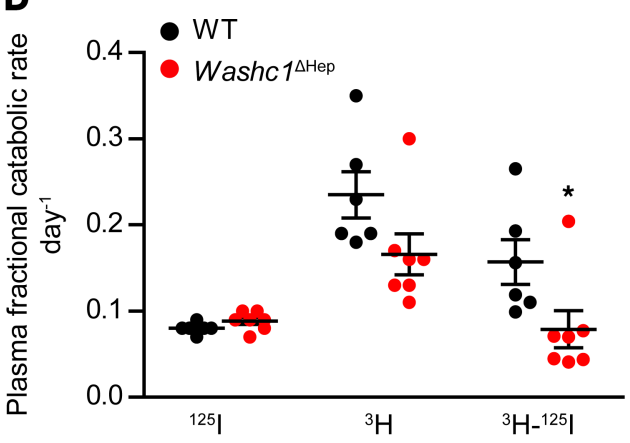

B $\bullet W T$

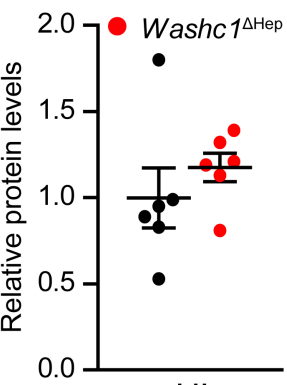

$\mathrm{HL}$

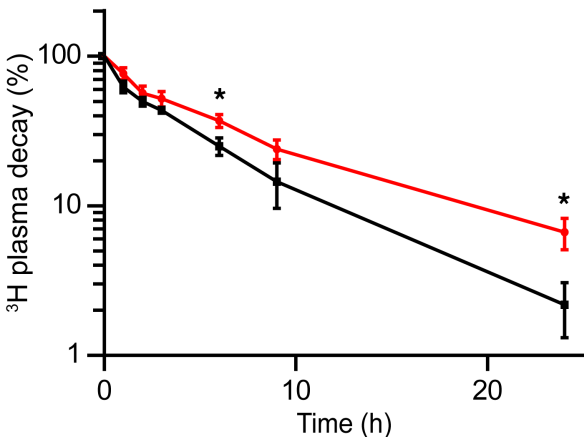

E

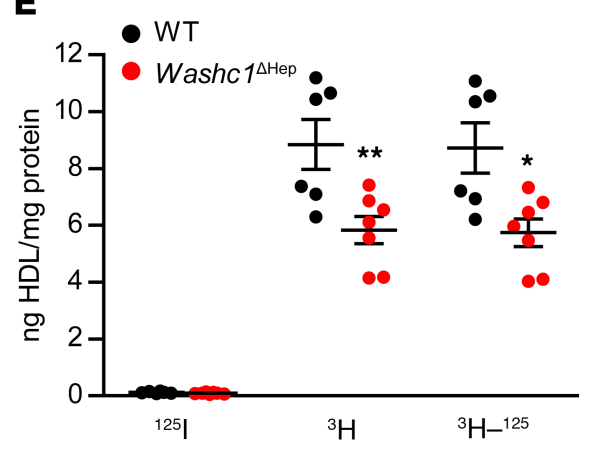

Figure 5. Hepatic uptake of HDL-C is impaired by reduced surface levels of SR-BI in hepatic WASH-deficient mice. (A) Immunoblot of whole-cell and cell-surface SR-BI levels in WT and Washc1 $1^{-/-}$primary hepatocytes. (B) Plasma hepatic lipase levels of male WT and Washc $1^{\text {Hep }}$ mice as determined by targeted proteomics $(n=6)$. (C) HDL protein ${ }^{125}$ I) and HDL-C $\left({ }^{3} \mathrm{H}\right)$ plasma decay in male WT and Washc1 $1^{\text {Hер }}$ mice $(n=6-7)$. (D) Fractional catabolic rate of plasma HDL holoparticle $\left({ }^{(25} \mathrm{I}\right), \mathrm{HDL}-\mathrm{C}\left({ }^{3} \mathrm{H}\right)$, and selective HDL-C clearance $\left({ }^{3} \mathrm{H}-{ }^{125} \mathrm{I}\right)$ of male WT and Washc $7^{1 \text { Hep }}$ mice. (E) HDL holoparticle $\left({ }^{125} \mathrm{I}\right)$, $\mathrm{HDL}-\mathrm{C}\left({ }^{3} \mathrm{H}\right)$, and selective HDL cholesterol uptake $\left({ }^{3} \mathrm{H}-125 \mathrm{I}\right)$ in liver of male WT and Washc ${ }^{1 \text { Hep }}$ mice $(n=6-7)$. Data are presented as the mean $\pm \mathrm{SEM},{ }^{*} P<0.05,{ }^{* *} P<0.01$, as determined by Student's $t$ test.

Furthermore, targeted proteomics analysis of FPLC fractions showed a leftward shift of ApoA-I and ApoE (Figure 6E), confirming that the HDL particles are also larger in Washc1/Commd1 ${ }^{\text {Hep }}$ mice (Figure 2, D-F). These in vivo data support the notion that the WASH and CCC complexes act together to form a crucial sorting pathway that is required to maintain normal plasma cholesterol levels.

IDOL- but not PCSK9-mediated LDLR degradation is exacerbated by WASH deficiency. The endocytic system is crucial for the LDLR degradation pathways mediated by either PCSK9 or IDOL, but the contribution of the WASH-CCC axis in these degradation pathways has yet to be defined. To this end, we assessed the effect of WASH deficiency on PCSK9- and IDOL-mediated LDLR degradation. WT and WASHC1-deficient MEFs (ref. 16 and Figure 7A) were incubated with recombinant human PCSK9 $(2.5 \mu \mathrm{g} / \mathrm{ml})$, and whole-cell extracts were harvested at different time points (Figure 7B). Immunoblots showed that PCSK9 decreased total LDLR levels in WT and WASHC1-deficient cells, but loss of WASHC1 did not affect the degradation kinetics of LDLR by PCSK9 (Figure 7, B and C).

Next, we incubated WT and WASHC1-deficient MEFs with the LXR agonist T0901317 (1 $\mu \mathrm{M}$ T09) to induce IDOL expression (47). Cells were collected at different intervals over an 8-hour period, and total 
A

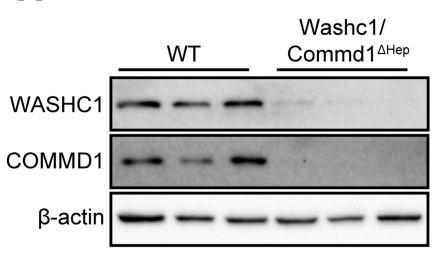

C

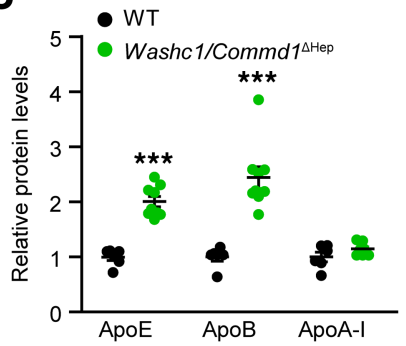

E

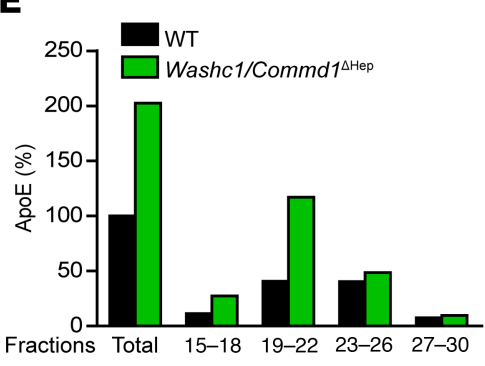

B

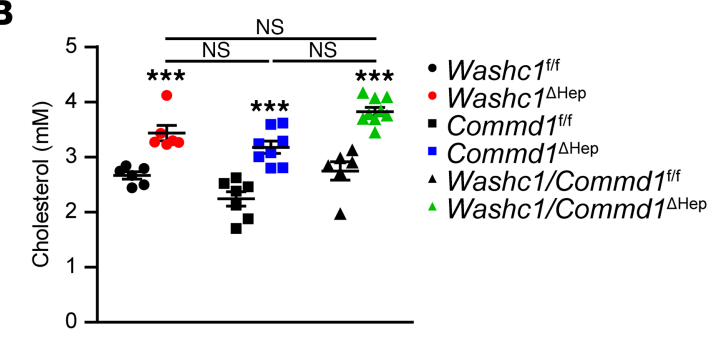

D
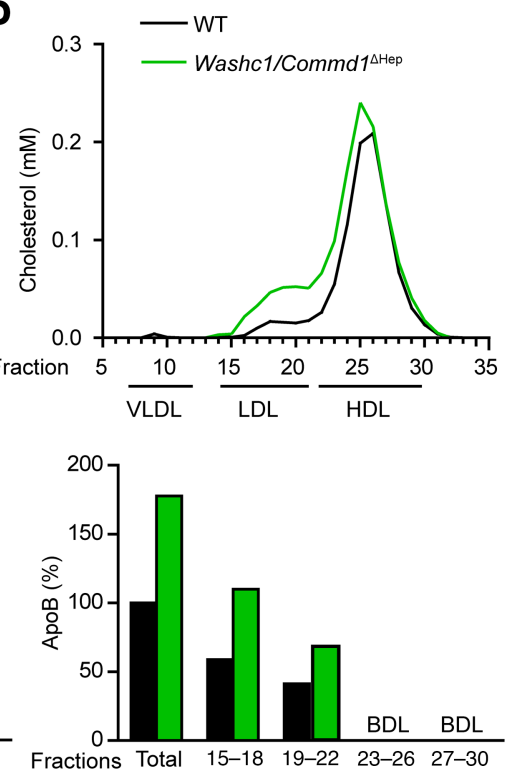

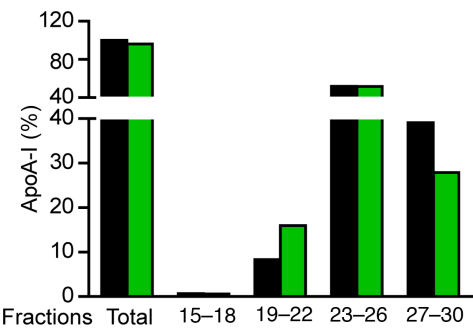

Figure 6. Characterization of Washc1/Commd14Hep mice. (A) WASHC1 and COMMD1 protein levels in livers of male Washc1/Commd1 ${ }^{\text {HHep }}$ and WT mice as determined by immunoblotting. (B) Total plasma cholesterol of male Washc1 ${ }^{\text {Hep }}$, Commd1 1Hер and Washc1/Commd1 ${ }^{\text {1Hep }}$ mice and their littermate controls (WT) ( $n=6-9)$. (C) Plasma apolipoprotein levels in male WT and Washc1/Commd1 1Hep mice determined by targeted proteomics, indicated by fold change vs WT littermate controls $(n=6-8)$. (D) Cholesterol levels of FPLC fractionated plasma pools of the experimental groups of mice. (E) Apolipoprotein levels in pooled FPLC fractionated plasma depicted in $\mathbf{D}$ as determined by targeted proteomics, indicated as percentage relative to the total apolipoprotein levels in these fractions. BLD, below detection limit. Data are presented as the mean \pm SEM, ${ }^{* *} P<0.001$ as determined by Student's $t$ test.

levels of LDLR were assessed by immunoblot analysis. As shown in Figure 7, D and E, loss of WASHC1 accelerated IDOL-mediated LDLR degradation. Taken together, these results suggest that the WASH complex participates in the IDOL-LDLR degradation pathway but not in the PCSK9-LDLR axis.

\section{Discussion}

Mutations in subunits of the WASH complex have been correlated with developmental and neurological defects in humans $(12,14,15,48)$. Here, we report that the WASH complex also has a pivotal role in the maintenance of plasma cholesterol levels. Our data indicate that WASH orchestrates the trafficking of the lipoprotein receptors LDLR and LRP1 as well as SR-BI to the plasma membrane for hepatic clearance of LDL-C and HDL-C, respectively.

The endolysosomal system consists of a series of organelles that is required to maintain cellular cholesterol and lipid homeostasis (49). In this article, we show that the WASH complex is also essential for the architecture of the endolysosomal system in hepatocytes, which is in line with previous observations in MEFs (4, 8-10). LDL-C bound to LDLR is hydrolyzed in the late endosomes/lysosomes (LEs/LYs) and, subsequently, exported from LEs/LYs by Niemann-Pick disease type C (NPC) proteins to the plasma membrane or endoplasmic reticulum (49). Loss of NPC impairs cholesterol export and enhances SREBP-2 activity, resulting in increased cholesterol biosynthesis and LDLR-mediated uptake and, eventually, in cholesterol accumulation in LEs/LYs $(50,51)$. Here, we found that the disturbed endolysosomal architecture in Washc $1^{1 \mathrm{Hep}}$ mice does not cause a defective intracellular cholesterol transport, considering that the protein levels of NPC1 (data not shown) and SREBP-2 activity were not changed upon Washcl ablation. In line with these findings, hepatic cholesterol 
A

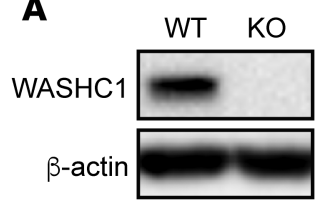

B

\begin{tabular}{|c|c|c|c|c|c|c|c|c|}
\hline \multirow[b]{2}{*}{ PCSK9 time $(\mathrm{h})$} & \multicolumn{4}{|c|}{ MEF WT } & \multicolumn{4}{|c|}{ MEF Washc1 KO } \\
\hline & 0 & 2 & 4 & 6 & 0 & 2 & 4 & 6 \\
\hline LDLR & $=$ & $=$ & $=$ & $=$ & $=$ & 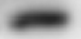 & $=$ & $=$ \\
\hline
\end{tabular}

D

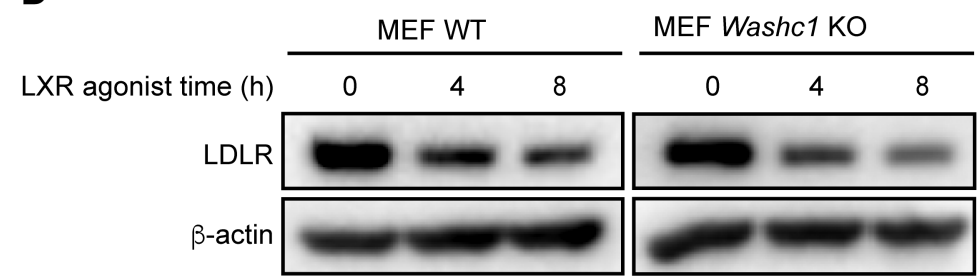

C
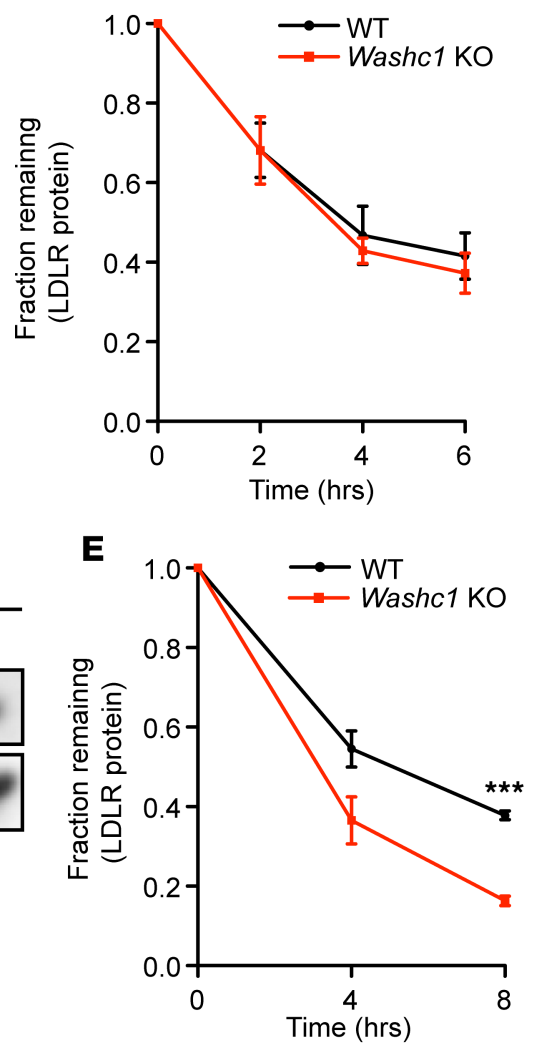

Figure 7. WASH deficiency increases IDOL-mediated LDLR degradation but did not affect the proteolysis of LDLR by PCSK9. (A) WASHC1 levels in WT and Washc1-knockout MEFs, as determined by immunoblotting. (B) MEFs were cultured in sterol-depleted medium for 24 hours and then incubated with recombinant human PCSK9 $(2.5 \mathrm{mg} / \mathrm{ml})$ for the indicated times. In the immunoblot analysis of LDLR, the blots represent 3 independent experiments. (C) Quantification of the time course of PCSK9-mediated LDLR degradation as shown in (B) $(n=3)$. (D) MEFs were cultured in sterol-depleted medium for 24 hours prior to T09 $(1 \mu \mathrm{M})$ incubation for the indicated times. Total LDLR protein levels were determined by immunoblot analysis, and the blots represent 3 independent experiments. (E) Quantification of LDLR levels relative to time point $\mathbf{O}$ hours $(n=3)$; the representative picture is shown in $\mathbf{D}$. Data are presented as the mean \pm SEM, ${ }^{* *} P<0.001$ as determined by Student's $t$ test.

content and hepatic cholesterol synthesis were not affected by WASH deficiency. Although hepatic LXR activity was slightly reduced upon Washcl ablation, this change did not translate into aberrant hepatic bile acid synthesis or biliary cholesterol and bile acid excretion during the investigation. Importantly, fecal neutral sterol and bile acid loss were unaffected in WASHC1-deficient mice, indicating unaffected whole-body cholesterol turnover. The lysosome is also an essential organelle in autophagy, a catabolic process important in maintaining hepatic and plasma TG levels and hepatic fatty acid oxidation (52). Interestingly, it has been shown that WASHC1 inhibits autophagic flux during mouse embryogenesis (53), but under the tested conditions, plasma and hepatic TG were not affected in hepatic WASHC1-deficient mice. Altogether, our data provide no evidence that hepatic WASHC1 deficiency results in dysfunctional lysosomes and aberrant intracellular cholesterol transport or cholesterol synthesis, despite their abnormal architecture.

In agreement with our in vitro studies (16), hepatocyte-specific WASH deficiency leads to reduced LDLR and LRP1 levels in the plasma membrane, which likely underlies elevated plasma LDL-C levels in $\mathrm{Washc}^{1^{\mathrm{Hep}}}$ mice. Interestingly, however, we also found an increase in HDL-C in Washc $1^{1 \mathrm{Hep}}$ mice. The diminished surface levels of SR-BI accompanied by reduced selective hepatic HDL-CE uptake suggest that the functioning of SR-BI-the main receptor for hepatic HDL-C uptake in mice $(54,55)$-is dependent on the WASH complex. The importance of hepatic SR-BI for HDL-C metabolism has also been shown in humans (56). Individuals who are heterozygous for a loss-of-function variant in SCARB1 (P376L), the gene encoding SR-BI, have increased levels of plasma HDL-C (56). Furthermore, common genetic variants near SCARB1 have been found to be correlated with plasma HDL-C levels $(57,58)$, underlying the importance of SR-BI in HDL-C metabolism in both species. 
Notably, in addition to increased plasma LDL-C levels, plasma HDL-C levels were also reported to be relatively high in a patient with RSS with a homozygous hypomorphic WASHC5 allele (16). Similar to the patient with RSS, both LDL-C and HDL-C are elevated in patients with X-linked intellectual disability caused by mutations in the gene encoding the CCC-complex component CCDC22 (16). Our current and previous mouse models mimic the dyslipidemic phenotype of patients with RSS and those with X-linked intellectual disability, as plasma LDL-C and HDL-C levels are both elevated upon either WASH or CCC ablation in mouse livers $(16,17)$, suggesting that both the CCC and WASH complexes are required for efficient hepatic LDL-C and HDL-C uptake in men and mice.

In vitro studies have shown that the CCC complex is physically associated with the WASH complex through the binding of the CCC components CCDC93 and CCDC22 to the WASH subunit WASHC2 (FAM21) (5). This interaction is required for the localization of the CCC complex to endosomes and subsequently for endosomal trafficking of integral membrane proteins. Furthermore, we have shown that loss of the CCC complex leads to increased expression of several WASH subunits, including WASHC1 (17). Here, we provide genetic evidence that the WASH and CCC complexes act in concert in the endosomal cargo recycling pathway, as inactivation of both complexes in mouse livers does not exacerbate the hypercholesterolemic phenotype.

The findings of our current study are particularly important, as very little is known about the mechanisms by which the endosomal trafficking of SR-BI is regulated. Several studies have shown that SR-BI is endocytosed $(59,60)$ and localized in recycling endosomes $(60)$. Our results indicate that the localization of SR-BI at the plasma membrane largely relies on the WASH-CCC axis. Based on the role of the WASH-CCC pathway in endosomal cargo recycling, we hypothesize that, after internalization, SR-BI is further transported to the endosomes from where it is directed back to the plasma membrane. The function of SR-BI being endocytosed and recycled is still unclear, as the selective uptake of HDL-CE by SR-BI is independent of the endocytosis of the entire HDL particle or SR-BI $(44,61,62)$. Similar observations were made in this study, in which an impairment of the WASH-CCC axis only led to reduced HDL-CE uptake without affecting ApoA-I clearance as well as its plasma levels, as expected in case of a reduction in the amount of SR-BI at the cell surface. It has been suggested, however, that SR-BI recycling might be involved in the retroendocytosis of HDL and facilitates transcytosis and transport of HDL-C into the bile canaliculus (60). Yet, we did not find any changes in biliary cholesterol excretion, which may suggest that a compensatory adaptive response is induced. It may well be that more significant conditions (e.g., blocking of endogenous cholesterol synthesis) are required to unmask a phenotype in this respect. Thus, our results and model might thus be relevant to obtain better insights into the biology of the intracellular trafficking pathway of SR-BI in cholesterol homeostasis.

The endolysosomal system is fundamental for the PCSK9- and IDOL-mediated degradation pathways (18-22). Here, we demonstrate that impaired endosomal LDLR recycling by the loss of WASHC1 does not affect PCSK9-mediated LDLR degradation. However, WASHC1 deficiency does accelerate LDLR proteolysis promoted by IDOL. These data indicate that the itinerary of PCSK9-LDLR to the lysosome is spatially separated from the LDLR recycling route $(11,23,63)$. On the other hand, LDLR targeted by IDOL for proteolysis might be transported to WASH-positive endosomes from where LDLR is further transported to the lysosome for degradation. Of note, it has been shown that endosomal sorting complexes required for transport machinery (ESCRT) is required for IDOL-mediated LDLR degradation $(19,20,22)$, and it has been proposed that ESCRT facilitates the transport of receptors into the lysosome that are not retrieved and, subsequently, recycled by the WASH-CCC axis $(11,23,63)$. Altogether, these data imply that the WASH complex participates in the sorting of ubiquitylated LDLR by IDOL to the lysosome.

Our data also unexpectedly suggest that overexpression of PCSK9 lowers hepatic LRP1 levels in Washc ${ }^{\Delta \mathrm{Hep}}$ mice. It has been shown that PCSK9 can bind to and decrease LRP1 in different cell lines (64). However, we and others could not confirm a role for PCSK9 in hepatic LRP1 degradation in WT mice, nor in hepatic COMMD1-deficient mice $(17,37,38)$. The underlying mechanism explaining our observation is unclear, but it has been speculated that the mammalian ATP-binding cassette transporter ABCA7 may have a role in stabilizing LRP1. ABCA7 is highly expressed in the liver (64), and it might be that disruption of the WASH complex impairs the protective function of ABCA7 in the stabilization of LRP1 and, hereby, sensitizes LRP1 for PCSK9-mediated degradation. Future experiments are needed to understand this observation at a molecular level.

In conclusion, this study extends the mechanistic basis for the increased plasma cholesterol levels in humans with defects in the WASH-CCC axis and appeal for extra medical attention with regard to plasma 
lipid levels and atherosclerotic cardiovascular disease risk. In addition, our work provides insights in the role of the WASH complex in IDOL- and PCSK9-mediated LDLR degradation.

\section{Methods}

Animals. Washc1 ${ }^{\mathrm{f} / \mathrm{f}}$ C57BL/6 mice (4) were crossed with Alb-Cre-expressing mice (The Jackson Laboratory, stock 003574) to generate liver-specific Washcl-knockout mice. The mice studied were between 12 and 20 weeks of age, and male mice were predominantly used for this study (except for the data shown in Supplemental Figure 2). The following primers were used for genotyping of the mice: floxed Washcl forward (5'-CGCATTGATCTTCCTATACGC-3') and reverse (3'-TGTCAGTCCTATGCTTAGTG-5'), Alb-Cre forward (5'-GCGGTCTGGCAGTAAAAACTATC-3'), and reverse (5'-ACGAACCTGGTCGAAATCAGTG-3'). The liver-specific Commd1-knockout mice have been generated as described previously (46). Mice lacking both COMMD1 and WASHC1 in the liver were obtained by intercrossing liver-specific Commd1- and Washc1-knockout mice.

To ablate hepatic LDLR expression of $W a s h c 1^{\Delta \mathrm{Hep}}$ mice and WT littermates, we expressed PCSK9-D377Y in the liver of these mice by retro-orbital injection of $3.0 \times 10^{11}$ vector genomes of AAV-PCSK9-D377Y, performed similar to that previously described (17). CETP expression was induced by injection of $5.0 \times 10^{11}$ Ad-CETP particles via the orbital vein adjusted to $100 \mu 1$ with sterile PBS. Ad-CETP virus was provided by Uwe Tietge (Department of Pediatrics, University of Groningen, University Medical Center Groningen, Groningen, the Netherlands).

All experiments have been performed with male mice $(n=4-7)$. Mice were housed individually and fed ad libitum with a standard rodent chow diet (RMH-B, AB Diets, the Netherlands). In all experiments, littermates were used as WT controls. Before sacrifice, mice were fasted for 4 hours. Tissues for mRNA and protein expression analysis were snap frozen in liquid nitrogen and stored at $-80^{\circ} \mathrm{C}$ until further analysis. Blood was drawn by cardiac puncture, and plasma was collected after centrifugation at $1,000 \mathrm{~g}$ for 10 minutes at $4^{\circ} \mathrm{C}$.

Tissue culture. WT and WASHC1-deficient mouse embryonic fibroblasts were cultured as described previously (16). Prior to PCSK9 (PC9-H5223, ACRO Biosystems) incubation (2.5 $\mu \mathrm{g} / \mathrm{ml})$, the MEFs were cultured in DMEM GlutaMAX with 2\% LPDS for 24 hours. MEFs were cultured for 24 hours in DMEM GlutaMAX supplemented with $2 \%$ lipoprotein-deficient serum, $100 \mu \mathrm{M}$ mevalonate, and $5 \mu \mathrm{g} / \mathrm{ml}$ of simvastatin before the LXR agonist T0901317 (T2320, MilliporeSigma) $(1 \mu \mathrm{M})$ was added to the media. Cells were harvested at the indicated time points for immunoblotting.

Immunoblotting. For immunoblotting, liver homogenates were obtained using NP40 buffer $(0.1 \%$ Nonidet P-40 [NP-40], 0.4 M NaCl, 10 mM Tris- $\mathrm{HCl}$ [pH 8.0], $1 \mathrm{mM}$ EDTA) supplemented with protease and phosphatase inhibitors (Roche). Protein concentration was determined using the Bradford assay (Bio-Rad). Thirty micrograms of protein was separated using SDS-PAGE and transferred to Amersham Hybond-P PVDF Transfer Membrane (GE Healthcare; RPN303F). Membranes were blocked in 5\% milk in Tris-buffered saline with $0.01 \%$ Tween 20 (MilliporeSigma) and incubated with the indicated antibodies. Proteins were visualized using a ChemiDoc XRS + System using Image Lab software version 5.2.1 (both from Bio-Rad).

Antibodies. A list of commercially bought antibodies is provided in Supplemental Table S2. Rabbit polyclonal antibodies against WASH1 (1:1000) (9), FAM21 (1:100) (9), CCDC53 (WASHC3, 1:1000) (65) and Strumpellin (WASHC5, 1:1000) (65) were previously described.

Transmission EM sample preparation and analysis. For EM analysis, mice were fasted for 18 hours and perfusion fixed with $4 \%$ formaldehyde in $0.1 \mathrm{M}$ phosphate buffer. The livers from the animals were rapidly removed, cut into small pieces $\left(\sim 2 \mathrm{~mm}^{3}\right)$, and placed and stored in glass vials with $4 \%$ formaldehyde until further processed. Samples were fixed at room temperature by adding Karnovsky fixative (2.5\% glutaraldehyde and $2 \%$ formaldehyde (Electron Microscopy Sciences) in $0.2 \mathrm{M}$ Cacodylate buffer, $\mathrm{pH}$ 7.4) for 2 hours at room temperature. They were then postfixed with $1 \%$ OsO4/1.5\% K3Fe(III)(CN)6 in $0.065 \mathrm{M}$ phosphate buffer for 2 hours at $4^{\circ} \mathrm{C}$ and finally for 1 hour with $0.5 \%$ uranyl acetate. After fixation, samples were dehydrated and embedded in Epon epoxy resin (Polysciences). Sixty-nanometer ultrathin sections were made and contrasted with uranyl acetate and lead citrate using the AC20 (Leica) and examined with a Technai T12 electron microscope (FEI Thermo Fischer). Images were taken from 2 different blocks per mouse and per condition. Twenty-five random images per block were collected for each mouse per condition (100 random images per condition).

All measurements on the lysosome size were taken using FiJi software. For the quantification of the lysosome area, the perimeters of lysosomes were selected one by one using the freehand selection tool in FiJi, and the encircled area was measured. For visualization of lysosomal tubulation, lysosomal perimeters were selected using the Lasso tool in Adobe Photoshop and the selected organelles were colored. 
FPLC. Plasma samples of each group of mice were pooled and fractionated by FPLC as previously described (66), with minor modifications. In brief, the system contained a PU-980 ternary pump with an LG-980-02 linear degasser and a UV-975 UV/VIS detector (Jasco). EDTA plasma was diluted 1:1 with Tris-buffered saline, and $300 \mu 1$ sample/buffer mixture was loaded onto a Superose 6 HR 10/300 column (GE Healthcare, Life Sciences Division) for lipoprotein separation at a flow rate of $0.5 \mathrm{ml} / \mathrm{min}$. TC and TG content of the fractions was determined as described in the information to follow.

Hepatic lipid extraction. Liver homogenates prepared as $15 \%(\mathrm{w} / \mathrm{v})$ solutions in PBS were subjected to lipid extraction according to the Bligh \& Dyer method (67). In short, $600 \mathrm{ml}$ of demi-water was mixed with $200 \mathrm{ml}$ of liver homogenate and $3 \mathrm{ml}$ of chloroform. After 30 minutes of incubation, $1.2 \mathrm{ml}$ of $\mathrm{H}_{2} \mathrm{O}$ and 1 $\mathrm{ml}$ of chloroform were added, mixed, and subsequently centrifuged (10 min, $500 \mathrm{~g}$ at room temperature). The chloroform layer was transferred into a new glass tube and was evaporated using nitrogen at $50^{\circ} \mathrm{C}$. Lipids were resolved in $1 \mathrm{ml}$ of chloroform and used for further determination of cholesterol and TG content.

Cholesterol and TG analysis in plasma and liver. TC levels were determined using colorimetric assays (11489232, Roche) with cholesterol standard FS (DiaSys Diagnostic Systems GmbH) as a reference. TG levels were measured using a Trig/GB kit (1187771, Roche) with Roche Precimat Glycerol standard (16658800) as a reference.

Targeted liquid chromatography-mass spectrometry analysis. To quantify the protein concentrations of ApoA1, ApoB, and ApoE in mouse plasma, we used a targeted proteomics assay as previously described (17). The protein levels of HL in nonheparinized plasma were quantified using the isotopically labeled standard peptide LSPDDANFVDAIHTFTR. Isotopically labeled standards were generated by concatenating all target peptides into synthetic proteins (Polyquant $\mathrm{GmbH}$ ) containing ${ }^{13} \mathrm{C}$-labeled lysines and arginines (so-called QconCATs). The known concentrations of these standard peptides were used to calculate the concentrations of the endogenous equivalents of the peptides (in amount of protein $[\mu \mathrm{g}$ or $\mathrm{mg}$ ] per volume [d1] plasma or FPLC fraction).

In-gel digestion and liquid chromatography-mass spectrometry (LC-MS) analyses were performed as described previously (17) with the following alterations: For plasma samples, $1 \mu 1$ plasma plus 20 ng QconCATs were loaded on the gel, and an equivalent of $100 \mathrm{nl}$ plasma plus $2 \mathrm{ng}$ QconCATs was injected in the LC-MS for analysis.

For the FPLC fractions, $15 \mu 1$ of the pooled FPLC fractions was loaded on the gel. The QconCATs were mixed after digestion, and the cleanup step after the digestion was excluded. An equivalent of a 3- $\mu 1$ sample plus 0.4 to $1 \mathrm{ng}$ QconCATs was injected in the LC-MS for analysis.

Gene expression analysis. One hundred milligrams of mouse liver was homogenized in $1 \mathrm{ml}$ QIAzol Lysis Reagent (Qiagen). Total RNA was isolated by chloroform extraction. Isopropanol-precipitated and ethanol-washed RNA pellets were dissolved in RNase/DNase-free water. One microgram of RNA was used to prepare cDNA with the Transcriptor Universal cDNA Master kit (Roche), according to the manufacturer's protocol. Twenty nanograms of cDNA was used for quantitative real-time PCR (qRT-PCR) analysis using the FastStart SYBR Green Master (Roche) and the 7900HT Fast Real-Time PCR System (Applied Biosystems). The PCR reaction was performed as follows: 2 minutes at $50^{\circ} \mathrm{C}, 10$ minutes at $95^{\circ} \mathrm{C}, 40$ cycles of 15 seconds at $95^{\circ} \mathrm{C}$, and 1 minute at $60^{\circ} \mathrm{C}$. Expression data were analyzed using SDS 2.3 software (Applied Biosystems), using the $\triangle \triangle C T$ method of calculation. PPIA expression was used as an internal control. The sequence of the primers is available upon request.

Biotinylation assay. Culture of primary hepatocytes and biotinylation assay were performed as previously described (38). Cells were washed 3 times with ice-cold PBS-CM buffer (PBS, 1-mM MgCl2, and $0.1 \mathrm{mM} \mathrm{CaCl} 2$ ). Subsequently, $0.5 \mathrm{mg} / \mathrm{ml}$ biotin reagent solution (EZ-Link Sulfo-NHS-SS Biotin, Thermo Scientific) in biotinylation buffer (10 $\mathrm{mM}$ triethanolamine, $\mathrm{pH} 8.0,150 \mathrm{mM} \mathrm{NaCl}$, and $2 \mathrm{mM} \mathrm{CaCl}$ ) was added to the cells for 30 minutes at $4^{\circ} \mathrm{C}$. The biotin reagent was removed, and cells were washed once with quenching buffer (PBS-CM, $25 \mathrm{mM}$ Tris- $\mathrm{HCl}, \mathrm{pH} 7.4$, and $192 \mathrm{mM}$ glycine) for 30 minutes at $4^{\circ} \mathrm{C}$. Next, cells were washed twice with PBS-CM and once with TBS-C (50 mM Tris-Cl, pH 7.4, 100 $\mathrm{mM} \mathrm{NaCl}$, and $2 \mathrm{mM} \mathrm{CaCl2})$. Cells were collected by scraping in TBS-C. Cells were centrifuged $(1,000$ $\mathrm{g}, 5 \mathrm{~min}, 4^{\circ} \mathrm{C}$ ), lysed in biotin lysis buffer ( $50 \mathrm{mM}$ Tris- $\mathrm{Cl}$, pH 7.4, $150 \mathrm{mM} \mathrm{NaCl}, 1 \% \mathrm{NP}-40,0.5 \%$ sodium deoxycholate, $5 \mathrm{mM}$ EDTA, and $5 \mathrm{mM}$ EGTA), incubated on ice for 15 minutes, and subsequently centrifuged for 15 minutes at 12,000 g. Protein concentration was determined: $30 \mathrm{mg}$ was used as input, $300 \mathrm{mg}$ was used diluted in biotin lysis buffer $(500 \mu \mathrm{l})$, and $30 \mu 1$ Neutravidin beads (Neutravidin plus ultralink beads, Thermo Scientific) were added and incubated for 4 hours at $4^{\circ} \mathrm{C}$. Beads were collected by 
centrifugation ( $500 \mathrm{~g}, 5 \mathrm{~min}$ ), washed 3 times with Biotin lysis buffer, once with high-salt buffer (50 mM Tris-Cl, pH 7.4 and $500 \mathrm{mM} \mathrm{NaCl}$ ), and once with low-salt buffer (10 mM Tris- $\mathrm{HCl})$. Finally, the beads were resuspended in $30 \mu 12 \times$ loading buffer.

Radioactive labeling of HDL. Total human HDL $(1.063<\mathrm{d}<1.21 \mathrm{~g} / \mathrm{ml})$ was obtained by sequential ultracentrifugation as described before (68). HDL was labeled with ${ }^{125} \mathrm{I}$ by a modification of the iodine monochloride method (69). To label HDL with ${ }^{3} \mathrm{H}$-cholesteryl-ether $\left({ }^{3} \mathrm{H}-\mathrm{CE}\right), 500 \mu \mathrm{Ci}$ of ${ }^{3} \mathrm{H}-\mathrm{CE}$ resuspended in $50 \mu \mathrm{l}$ of ethanol was added to a solution containing heat-inactivated human lipoprotein-deficient serum (200 mg protein) and iodinated HDL (6 mg protein). Radiolabeled HDL was incubated overnight at $37^{\circ} \mathrm{C}$ with gentle shaking, followed by re-isolation of the labeled HDL by sequential ultracentrifugation. Animals were injected with $8333 \mathrm{~Bq}{ }^{3} \mathrm{H}-\mathrm{CE}$ with an activity of $357 \mathrm{~Bq} / \mu \mathrm{g} \mathrm{HDL}$ and $33333 \mathrm{~Bq}{ }^{125} \mathrm{I}-\mathrm{HDL}$ with an activity of $2465 \mathrm{~Bq} / \mu \mathrm{g} \mathrm{HDL}$.

$V L D L$ production assay. After a 4-hour morning fasting, mice were injected i.p. with $100 \mathrm{mg} / \mathrm{ml}$ poloxamer 407 (BASF) in PBS (1 g per kg body weight). Blood was drawn by retro-orbital puncture at time points 0, 30, 60, 120, and 240 minutes. Plasma samples were used for TG determination.

Determination of cholesterol synthesis in vivo. $\left[{ }^{13} \mathrm{C}\right]$-acetate was added to drinking water (99 atom \%, Isotec/MilliporeSigma) for 3 days. Every 12 hours, a blood sample was taken via tail bleeding on filter paper to determine fractional cholesterol synthesis rates. Total cholesterol (TC) was extracted from blood spots using ethanol/acetone $(1: 1 \mathrm{v} / \mathrm{v})$. Cholesterol synthesis measurements were performed as previously described (70).

Fecal and biliary bile acid and cholesterol measurements. Feces were collected over a period of 24 hours. Mice were anesthetized by i.p. injection of Hypnorm ( $1 \mathrm{ml} / \mathrm{kg}$ ) (fentanylcitrate $0.315 \mathrm{mg} / \mathrm{ml}$ and fluanisone $10 \mathrm{mg}$ / $\mathrm{ml}$, VetaPharma) and diazepam (10 mg/kg) (Centrafarm) and subjected to gallbladder cannulation for $20 \mathrm{~min}$ utes as described previously (71). During bile collection, body temperature was stabilized using an incubator. Bile was stored at $-20^{\circ} \mathrm{C}$ until analyzed. Cholesterol and bile acids were measured as described earlier $(72,73)$.

Statistics. In vitro data were obtained from 3 independent experiments \pm SEM. Mouse data show average values $\pm \operatorname{SEM}(n=4-7)$. Analyses were performed using GraphPad version 6.05 (GraphPad software). Unpaired 2-tailed Student's $t$ test was used to test for statistical significance between 2 groups. For all experiments, a $P$ value of $<0.05$ was considered statistically significant.

Study approval. All animal studies were approved by the Institutional Animal Care and Use Committee, University of Groningen (Groningen, the Netherlands).

\section{Author contributions}

MW and BVDS designed the studies. PZ assisted in the design and the analysis of the selective HDLCE uptake study. MW, PZ, DYV, MYJ, and SW conducted the experiments with the assistance from MS, NH, DD, and NK. YTV and JCW performed and analyzed the proteomics experiments. NL and JK generated and analyzed the EM data. TH and FK analyzed the bile acid and cholesterol flux experiments. DB provided the conditional Washc1-knockout model, WT, and WASHC1-deficient MEFs and antibodies. MW, JAK, and BVDS analyzed and interpreted the data and wrote the manuscript with the contributions from all authors.

\section{Acknowledgments}

The authors gratefully acknowledge Rene van Tienhoven, Rick Havinga, and Martijn Koehorst for their technical assistance. We also acknowledge Mirjam Koster, Sameh Youssef, and Alain de Bruin from the Dutch Molecular Pathology Center (Department of Pathobiology, Faculty of Veterinary Medicine, Utrecht University, Utrecht, the Netherlands) for their pathological analysis of the liver sections. This research was financially supported by the Netherlands Organization for Scientific Research (NWO-ALW grant no. 817.02.022 to BVDS), EU FP7 project TransCard (FP7-603091-2), Genius II (CVON 2017-20, the Netherlands Heart Foundation), the Jan Kornelis de Cock Stichting, the Graduate School for Drug Exploration (GUIDE), and the University of Groningen. JAK is an established investigator of the Netherlands Heart Foundation (2015T068).

Address correspondence to: Bart van de Sluis, Antonius Deusinglaan 1, 9713 AV, Groningen, the Netherlands. Phone: 31.50.361.6793; Email: a.j.a.van.de.sluis@umcg.nl. 
1. Seaman MN, Gautreau A, Billadeau DD. Retromer-mediated endosomal protein sorting: all WASHed up! Trends Cell Biol. 2013;23(11):522-528.

2. Wang J, Fedoseienko A, Chen B, Burstein E, Jia D, Billadeau DD. Endosomal receptor trafficking: retromer and beyond. Traffic. 2018;19(8):578-590.

3. Alekhina O, Burstein E, Billadeau DD. Cellular functions of WASP family proteins at a glance. J Cell Sci. 2017;130(14):2235-2241.

4. Gomez TS, Gorman JA, de Narvajas AA, Koenig AO, Billadeau DD. Trafficking defects in WASH-knockout fibroblasts originate from collapsed endosomal and lysosomal networks. Mol Biol Cell. 2012;23(16):3215-3228.

5. Phillips-Krawczak CA, et al. COMMD1 is linked to the WASH complex and regulates endosomal trafficking of the copper transporter ATP7A. Mol Biol Cell. 2015;26(1):91-103.

6. Temkin P, Lauffer B, Jäger S, Cimermancic P, Krogan NJ, von Zastrow M. SNX27 mediates retromer tubule entry and endosome-to-plasma membrane trafficking of signalling receptors. Nat Cell Biol. 2011;13(6):715-721.

7. Puthenveedu MA, et al. Sequence-dependent sorting of recycling proteins by actin-stabilized endosomal microdomains. Cell. 2010;143(5):761-773.

8. Derivery E, Sousa C, Gautier JJ, Lombard B, Loew D, Gautreau A. The Arp2/3 activator WASH controls the fission of endosomes through a large multiprotein complex. Dev Cell. 2009;17(5):712-723.

9. Gomez TS, Billadeau DD. A FAM21-containing WASH complex regulates retromer-dependent sorting. Dev Cell. 2009;17(5):699-711.

10. Duleh SN, Welch MD. WASH and the Arp2/3 complex regulate endosome shape and trafficking. Cytoskeleton (Hoboken). 2010;67(3):193-206.

11. McNally KE, Cullen PJ. Endosomal retrieval of cargo: retromer is not alone. Trends Cell Biol. 2018;28(10):807-822.

12. Ropers F, et al. Identification of a novel candidate gene for non-syndromic autosomal recessive intellectual disability: the WASH complex member SWIP. Hum Mol Genet. 2011;20(13):2585-2590.

13. Valdmanis PN, et al. Mutations in the KIAA0196 gene at the SPG8 locus cause hereditary spastic paraplegia. Am J Hum Genet. 2007;80(1):152-161

14. de Bot ST, et al. Pure adult-onset spastic paraplegia caused by a novel mutation in the KIAA0196 (SPG8) gene. J Neurol. 2013;260(7):1765-1769.

15. Elliott AM, et al. A novel mutation in KIAA0196: identification of a gene involved in Ritscher-Schinzel/3C syndrome in a First Nations cohort. J Med Genet. 2013;50(12):819-822.

16. Bartuzi P, et al. CCC- and WASH-mediated endosomal sorting of LDLR is required for normal clearance of circulating LDL. Nat Commun. 2016;7:10961.

17. Fedoseienko A, et al. The COMMD family regulates plasma LDL levels and attenuates atherosclerosis through stabilizing the CCC complex in endosomal LDLR trafficking. Circ Res. 2018;122(12):1648-1660.

18. Wang Y, Huang Y, Hobbs HH, Cohen JC. Molecular characterization of proprotein convertase subtilisin/kexin type 9-mediated degradation of the LDLR. J Lipid Res. 2012;53(9):1932-1943.

19. Scotti E, et al. IDOL stimulates clathrin-independent endocytosis and multivesicular body-mediated lysosomal degradation of the low-density lipoprotein receptor. Mol Cell Biol. 2013;33(8):1503-1514.

20. Sorrentino V, et al. The LXR-IDOL axis defines a clathrin-, caveolae-, and dynamin-independent endocytic route for LDLR internalization and lysosomal degradation. J Lipid Res. 2013;54(8):2174-2184.

21. Horton JD, Cohen JC, Hobbs HH. PCSK9: a convertase that coordinates LDL catabolism. J Lipid Res. 2009;50(supp1):S172-S177.

22. Pietiäinen V, et al. NDRG1 functions in LDL receptor trafficking by regulating endosomal recycling and degradation. $J$ Cell Sci. 2013;126(pt 17):3961-3971.

23. McNally KE, et al. Retriever is a multiprotein complex for retromer-independent endosomal cargo recycling. Nat Cell Biol. 2017;19(10):1214-1225.

24. Harbour ME, Breusegem SY, Seaman MN. Recruitment of the endosomal WASH complex is mediated by the extended 'tail' of Fam21 binding to the retromer protein Vps35. Biochem J. 2012;442(1):209-220.

25. Jia D, Gomez TS, Billadeau DD, Rosen MK. Multiple repeat elements within the FAM21 tail link the WASH actin regulatory complex to the retromer. Mol Biol Cell. 2012;23(12):2352-2361.

26. Harbour ME, Breusegem SY, Antrobus R, Freeman C, Reid E, Seaman MN. The cargo-selective retromer complex is a recruiting hub for protein complexes that regulate endosomal tubule dynamics. J Cell Sci. 2010;123(Pt 21):3703-3717.

27. Koo C, Innerarity TL, Mahley RW. Obligatory role of cholesterol and apolipoprotein E in the formation of large cholesterol-enriched and receptor-active high density lipoproteins. J Biol Chem. 1985;260(22):11934-11943.

28. Yamashita S, Sprecher DL, Sakai N, Matsuzawa Y, Tarui S, Hui DY. Accumulation of apolipoprotein E-rich high density lipoproteins in hyperalphalipoproteinemic human subjects with plasma cholesteryl ester transfer protein deficiency. J Clin Invest. 1990;86(3):688-695.

29. Lee SX, et al. FoxO transcription factors are required for hepatic HDL cholesterol clearance. J Clin Invest. 2018;128(4):1615-1626.

30. Guyard-Dangremont V, Desrumaux C, Gambert P, Lallemant C, Lagrost L. Phospholipid and cholesteryl ester transfer activities in plasma from 14 vertebrate species. Relation to atherogenesis susceptibility. Comp Biochem Physiol B, Biochem Mol Biol. 1998;120(3):517-525.

31. Gordon SM, Li H, Zhu X, Shah AS, Lu LJ, Davidson WS. A comparison of the mouse and human lipoproteome: suitability of the mouse model for studies of human lipoproteins. J Proteome Res. 2015;14(6):2686-2695.

32. Ishibashi S, Brown MS, Goldstein JL, Gerard RD, Hammer RE, Herz J. Hypercholesterolemia in low density lipoprotein receptor knockout mice and its reversal by adenovirus-mediated gene delivery. J Clin Invest. 1993;92(2):883-893.

33. Marotti KR, Castle CK, Murray RW, Rehberg EF, Polites HG, Melchior GW. The role of cholesteryl ester transfer protein in primate apolipoprotein A-I metabolism. Insights from studies with transgenic mice. Arterioscler Thromb. 1992;12(6):736-744.

34. Harder CJ, Meng A, Rippstein P, McBride HM, McPherson R. SR-BI undergoes cholesterol-stimulated transcytosis to the bile canaliculus in polarized WIF-B cells. J Biol Chem. 2007;282(2):1445-1455.

35. Rohlmann A, Gotthardt M, Hammer RE, Herz J. Inducible inactivation of hepatic LRP gene by cre-mediated recombination confirms role of LRP in clearance of chylomicron remnants. J Clin Invest. 1998;101(3):689-695. 
36. Bjørklund MM, et al. Induction of atherosclerosis in mice and hamsters without germline genetic engineering. Circ Res. 2014;114(11):1684-1689.

37. Fu T, Guan Y, Xu J, Wang Y. APP, APLP2 and LRP1 interact with PCSK9 but are not required for PCSK9-mediated degradation of the LDLR in vivo. Biochim Biophys Acta Mol Cell Biol Lipids. 2017;1862(9):883-889.

38. Lagace TA, et al. Secreted PCSK9 decreases the number of LDL receptors in hepatocytes and in livers of parabiotic mice. $J$ Clin Invest. 2006;116(11):2995-3005.

39. Acton S, Rigotti A, Landschulz KT, Xu S, Hobbs HH, Krieger M. Identification of scavenger receptor SR-BI as a high density lipoprotein receptor. Science. 1996;271(5248):518-520.

40. Amar MJ, et al. Hepatic lipase facilitates the selective uptake of cholesteryl esters from remnant lipoproteins in apoE-deficient mice. J Lipid Res. 1998;39(12):2436-2442.

41. Dugi KA, et al. In vivo evidence for both lipolytic and nonlipolytic function of hepatic lipase in the metabolism of HDL. Arterioscler Thromb Vasc Biol. 2000;20(3):793-800.

42. Brundert M, Heeren J, Greten H, Rinninger F. Hepatic lipase mediates an increase in selective uptake of HDL-associated cholesteryl esters by cells in culture independent from SR-BI. J Lipid Res. 2003;44(5):1020-1032.

43. Dichek HL, et al. Overexpression of hepatic lipase in transgenic mice decreases apolipoprotein B-containing and high density lipoproteins. Evidence that hepatic lipase acts as a ligand for lipoprotein uptake. J Biol Chem. 1998;273(4):1896-1903.

44. Glass C, Pittman RC, Weinstein DB, Steinberg D. Dissociation of tissue uptake of cholesterol ester from that of apoprotein A-I of rat plasma high density lipoprotein: selective delivery of cholesterol ester to liver, adrenal, and gonad. Proc Natl Acad Sci U S A 1983;80(17):5435-5439.

45. Pittman RC, Steinberg D. Sites and mechanisms of uptake and degradation of high density and low density lipoproteins. J Lipid Res. 1984;25(13):1577-1585.

46. Vonk WI, et al. Liver-specific Commd1 knockout mice are susceptible to hepatic copper accumulation. PLoS One. 2011;6(12):e29183.

47. Hong $\mathrm{C}$, et al. The $\mathrm{E} 3$ ubiquitin ligase IDOL induces the degradation of the low density lipoprotein receptor family members VLDLR and ApoER2. J Biol Chem. 2010;285(26):19720-19726.

48. Kolanczyk M, et al. Missense variant in CCDC22 causes X-linked recessive intellectual disability with features of RitscherSchinzel/3C syndrome. Eur J Hum Genet. 2015;23(5):720.

49. Ikonen E. Cellular cholesterol trafficking and compartmentalization. Nat Rev Mol Cell Biol. 2008;9(2):125-138.

50. Ikonen E. Mechanisms of cellular cholesterol compartmentalization: recent insights. Curr Opin Cell Biol. 2018;53:77-83.

51. Luo J, Jiang LY, Yang H, Song BL. Intracellular cholesterol transport by sterol transfer proteins at membrane contact sites. Trends Biochem Sci. 2019;44(3):273-292.

52. Ward C, et al. Autophagy, lipophagy and lysosomal lipid storage disorders. Biochim Biophys Acta. 2016;1861(4):269-284

53. Xia P, et al. WASH inhibits autophagy through suppression of Beclin 1 ubiquitination. EMBO J. 2013;32(20):2685-2696.

54. Huby T, et al. Knockdown expression and hepatic deficiency reveal an atheroprotective role for SR-BI in liver and peripheral tissues. J Clin Invest. 2006;116(10):2767-2776.

55. Zhang Y, Da Silva JR, Reilly M, Billheimer JT, Rothblat GH, Rader DJ. Hepatic expression of scavenger receptor class B type I (SR-BI) is a positive regulator of macrophage reverse cholesterol transport in vivo. J Clin Invest. 2005;115(10):2870-2874.

56. Zanoni P, et al. Rare variant in scavenger receptor BI raises HDL cholesterol and increases risk of coronary heart disease. Science. 2016;351(6278):1166-1171.

57. Teslovich TM, et al. Biological, clinical and population relevance of 95 loci for blood lipids. Nature. 2010;466(7307):707-713.

58. Vergeer M, et al. Genetic variant of the scavenger receptor BI in humans. N Engl J Med. 2011;364(2):136-145.

59. Pagler TA, et al. SR-BI-mediated high density lipoprotein (HDL) endocytosis leads to HDL resecretion facilitating cholesterol efflux. J Biol Chem. 2006;281(16):11193-11204.

60. Silver DL, Wang N, Xiao X, Tall AR. High density lipoprotein (HDL) particle uptake mediated by scavenger receptor class B type 1 results in selective sorting of HDL cholesterol from protein and polarized cholesterol secretion. J Biol Chem. 2001;276(27):25287-25293.

61. Nieland TJ, Ehrlich M, Krieger M, Kirchhausen T. Endocytosis is not required for the selective lipid uptake mediated by murine SR-BI. Biochim Biophys Acta. 2005;1734(1):44-51.

62. Quarfordt S, Hanks J, Jones RS, Shelburne F. The uptake of high density lipoprotein cholesteryl ester in the perfused rat liver. J Biol Chem. 1980;255(7):2934-2937.

63. Cullen PJ, Steinberg F. To degrade or not to degrade: mechanisms and significance of endocytic recycling. Nat Rev Mol Cell Biol. 2018;19(11):679-696.

64. Canuel M, Sun X, Asselin MC, Paramithiotis E, Prat A, Seidah NG. Proprotein convertase subtilisin/kexin type 9 (PCSK9) can mediate degradation of the low density lipoprotein receptor-related protein 1 (LRP-1). PLoS One. 2013;8(5):e64145.

65. Jia D, et al. WASH and WAVE actin regulators of the Wiskott-Aldrich syndrome protein (WASP) family are controlled by analogous structurally related complexes. Proc Natl Acad Sci U S A. 2010;107(23):10442-10447.

66. Gerdes LU, Gerdes C, Klausen IC, Faergeman O. Generation of analytic plasma lipoprotein profiles using two prepacked superose 6B columns. Clin Chim Acta. 1992;205(1-2):1-9.

67. Bligh EG, Dyer WJ. A rapid method of total lipid extraction and purification. Can J Biochem Physiol. 1959;37(8):911-917.

68. Hatch FT. Practical methods for plasma lipoprotein analysis. Adv Lipid Res. 1968;6:1-68.

69. Tietge UJ, et al. Overexpression of secretory phospholipase A(2) causes rapid catabolism and altered tissue uptake of high density lipoprotein cholesteryl ester and apolipoprotein A-I. J Biol Chem. 2000;275(14):10077-10084.

70. Renfurm LN, et al. Cholesterol synthesis and de novo lipogenesis in premature infants determined by mass isotopomer distribution analysis. Pediatr Res. 2004;56(4):602-607.

71. Kuipers F, et al. Altered lipid metabolism in apolipoprotein E-deficient mice does not affect cholesterol balance across the liver. Hepatology. 1996;24(1):241-247.

72. Hulzebos CV, et al. Measurement of parameters of cholic acid kinetics in plasma using a microscale stable isotope dilution technique: application to rodents and humans. J Lipid Res. 2001;42(11):1923-1929.

73. Kok T, et al. Enterohepatic circulation of bile salts in farnesoid X receptor-deficient mice: efficient intestinal bile salt absorption in the absence of ileal bile acid-binding protein. J Biol Chem. 2003;278(43):41930-41937. 